\title{
Un nuevo catálogo de terremotos para la República de Nicaragua
}

\author{
A new earthquake catalog for Nicaragua Republic
}

\author{
Historial del artículo \\ Recibido: \\ 24 de septiembre de 2021 \\ Revisado \\ 7 de noviembre de 2021 \\ Aceptado: \\ 17 de noviembre de 2021
}

\section{Leonardo Álvarez ${ }^{\mathrm{a}}$}

${ }^{a}$ Ministerio de Transporte e Infraestructura, Managua, Nicaragua. Correo electrónico: leoalvar50@gmail.com. ORCID: https:/l orcid.org/0000-0002-7406-6614

\section{Palabras clave}

Catálogo de terremotos, ley de Omori, modelo ETAS, sismicidad

\section{Keywords}

Earthquake catalog, ETAS model, Omori's law, seismicity

\section{Resumen}

Se presenta un nuevo catálogo de terremotos de Nicaragua confeccionado a partir de todas las fuentes disponibles, su unificación a magnitud MW, la extracción de los agrupamientos más importantes en la depresión de Nicaragua y la preparación de una variante sin eventos relacionados. El catálogo consta de 76.935 terremotos de los cuales 74.500 tienen al menos una determinación de magnitud. Se analizan los parámetros del régimen sísmico (a,b,Mmax) en siete zonas delimitadas convencionalmente. Se determinan las características estadísticas de los agrupamientos seleccionados (ajuste de los modelos ETAS y Omori modificado). Se analiza el comportamiento temporal de la liberación de energía en dos zonas (subducción Pacífico y depresión de Nicaragua) y se preparan para ambos los gráficos espacio temporales a partir del inicio del siglo XX. Se realizan estimados de los lugares de ocurrencia de eventos fuertes en el futuro mediato y se estima su magnitud máxima. Las Mmax posibles son de 6,5 para las zonas en la depresión de Nicaragua y 7,8 en la zona de subducción del Pacífico.

\section{Abstract}

A new catalog of earhquakes for Nicaragua seismicity is presented. It was prepared from all available sources, unified to MW magnitude, and declustered fom related events. The catalog comprises 76.935 earthquakes and from these 74.500 have at least one magitude determination. The parameters of seismic regime (a,b,Mmax) of 7 zones, conventionaly delimited, are analized. The statistical characteristics of selected clusters in Nicaraguan Depression are determined (adjustment of ETAS and Modified Omori models). The temporal behavior of energy release in two zones (Pacific subduction and Nicaraguan Depression) is analized, and for both zones are prepared the space-time plots from the beginning of XX Century. Assessment of the places of ocurrence of strong earthquakes in mediate future is performed for both zones and its maximum magnitudes are estimated. The posible Mmax are of 6,5 in Nicaraguan Depression and 7,8 in subduction zones of the Pacific. 


\section{Introducción}

La sismicidad de Nicaragua está documentada desde el siglo XVI, cuando los conquistadores españoles reportaron el primer sismo de que se tiene noticia en 1520. Se han reportados numerosos sismos con grandes afectaciones, los más conocidos son el de Managua de diciembre de 1972, que destruyó virtualmente toda la ciudad, y el de 1992, clasificado como sismo "lento" (prácticamente sin oscilaciones de alta frecuencia) seguido de un gran tsunami que agarró desprevenidos a los habitantes de una amplia zona costera (Kanamori \& Kikuchi, 1993). Los intentos de sistematizar los estudios de la sismicidad de Nicaragua comienzan con posterioridad al sismo de 1972, orientándose a la amenaza sísmica, el efecto de sitio y la instalación de estaciones sismológicas locales. Antes de ello solo se publicaban mapas aislados de epicentros.

La base de todo estudio de sismicidad y estimación de la amenaza sísmica la constituye un catálogo de terremotos. Es por ello que abundan tales estudios. Algunos se limitan a estudiar con un elevado grado de detalle la ocurrencia de terremotos en períodos reducidos (Cheng et al., 2017; Lolli et al., 2020), trabajos orientados a estudios específicos de sismicidad. Otros trabajos tratan de abarcar toda la historia sísmica de una región y están dirigidos a realizar estudios de peligrosidad sísmica fundamentalmente (Beauval et al., 2013; Brax et al., 2019). Su preparación requiere la consulta de diferentes fuentes y el establecimiento de criterios de selección, entre la información disponible, de la más confiable. En diferentes momentos se han confeccionado para Nicaragua catálogos de uso limitado que se discuten más adelante. En este trabajo se describe el proceso usado para confeccionar un nuevo catálogo de Nicaragua, mucho más general, y cualitativamente diferente a los anteriores.

Un terremoto se caracteriza por una serie de parámetros fundamentales, como son las coordenadas geográficas (grado y fracción) del epicentro, su profundidad, el tiempo de origen GMT (hora del meridiano de Greenwich) y determinaciones de magnitud (una medida de la energía liberada). Antes de la etapa de registro instrumental, los terremotos se caracterizaban en términos de la intensidad sísmica (medición de efectos sobre personas, construcciones y medio ambienta) y su conversión a magnitud debe hacerse mediante el análisis de la distribución espacial de la intensidad. A esta magnitud le llamamos $\mathrm{M}_{\mathrm{r}}$. Los registros instrumentales permiten realizar estimaciones de magnitud más precisas mediante la medición de amplitudes y períodos de algunas ondas, así como de la duración del registro, siendo las más conocidas la $\mathrm{m}_{\mathrm{B}}$ (en base a ondas internas $\mathrm{P}$ de corto período), la $\mathrm{M}_{\mathrm{S}}$ (en base a ondas superficiales) y la $\mathrm{M}_{\mathrm{D}}$ (en base a la duración de registro). También es habitual encontrar magnitudes locales determinadas por diferentes métodos, y que se simbolizan por $\mathrm{M}_{\mathrm{L}}$. Adicionalmente existe una magnitud que se determina a partir del espectro de la onda $\mathrm{P}$, y es llamada "magnitud de momento" $\mathrm{M}_{\mathrm{w}}$. Esta última magnitud resulta muy estable dentro de todo el rango energético medible de los terremotos y los catálogos de terremotos tienden cada vez más a estar homogenizados en términos de ella (Cheng et al., 2017). La determinación de coordenadas y tiempo de origen ha pasado por diferentes etapas; primeramente una forma aproximada por distribución de intensidades sentidas (para el período anterior al registro instrumental), seguida por la determinación de hipocentros (epicentro y profundidad) usando tiempos de llegada de diferentes ondas y tablas de tiempo de recorrido para dichas ondas previamente calculadas, y finalmente, a partir de la segunda mitad del siglo Xx, por la aplicación de métodos numéricos al proceso (permitiendo el cálculo de errores) usando como datos los tiempos de llegada en las estaciones y modelos de corteza. Una explicación detallada de todos estos procesos puede verse en (Bormann, 2012).

La mayoría de los catálogos de terremotos existentes generalmente tienen un número limitado de estimaciones de magnitud, limitándose a las más habituales, generalmente $\mathrm{m}_{\mathrm{B}}, \mathrm{M}_{\mathrm{S}} \mathrm{y}_{\mathrm{w}}$, y en algunos casos añadiendo alguna magnitud local, estando presentes casos de magnitudes "transformadas" de las originales determinadas en las estaciones. Fue un objetivo fundamental en este trabajo incluir en el nuevo catálogo hasta doce magnitudes diferentes, lo que permite tomar en consideración las determinaciones de una misma magnitud por diferentes agencias, así como magnitudes determinadas por cualquier método. Esto garantiza poder usarlo para cualquier estudio posterior. La idea subyacente es que nuevas investigaciones sobre la ocurrencia de terremotos no influyan grandemente sobre las características del catálogo. El criterio fundamental fue utilizar el máximo posible de fuentes para cada terremoto, seleccionar coordenadas y tiempo de origen de la "más confiable" y recolectar la totalidad de estimaciones de magnitud realizadas en cada caso. El proceso de discriminación de los datos y confección de un registro único por terremoto conllevó a realizar un trabajo de recolección de fuentes primarias de datos, selección de un formato en que se pudiera incluir el máximo posible de información por terremoto y confección de numerosos programas de conversión de formato, comparación de datos, manejo de información en una base de datos, representación gráfica, etc. Se consideró todo el diapasón de magnitudes posible, lo que 


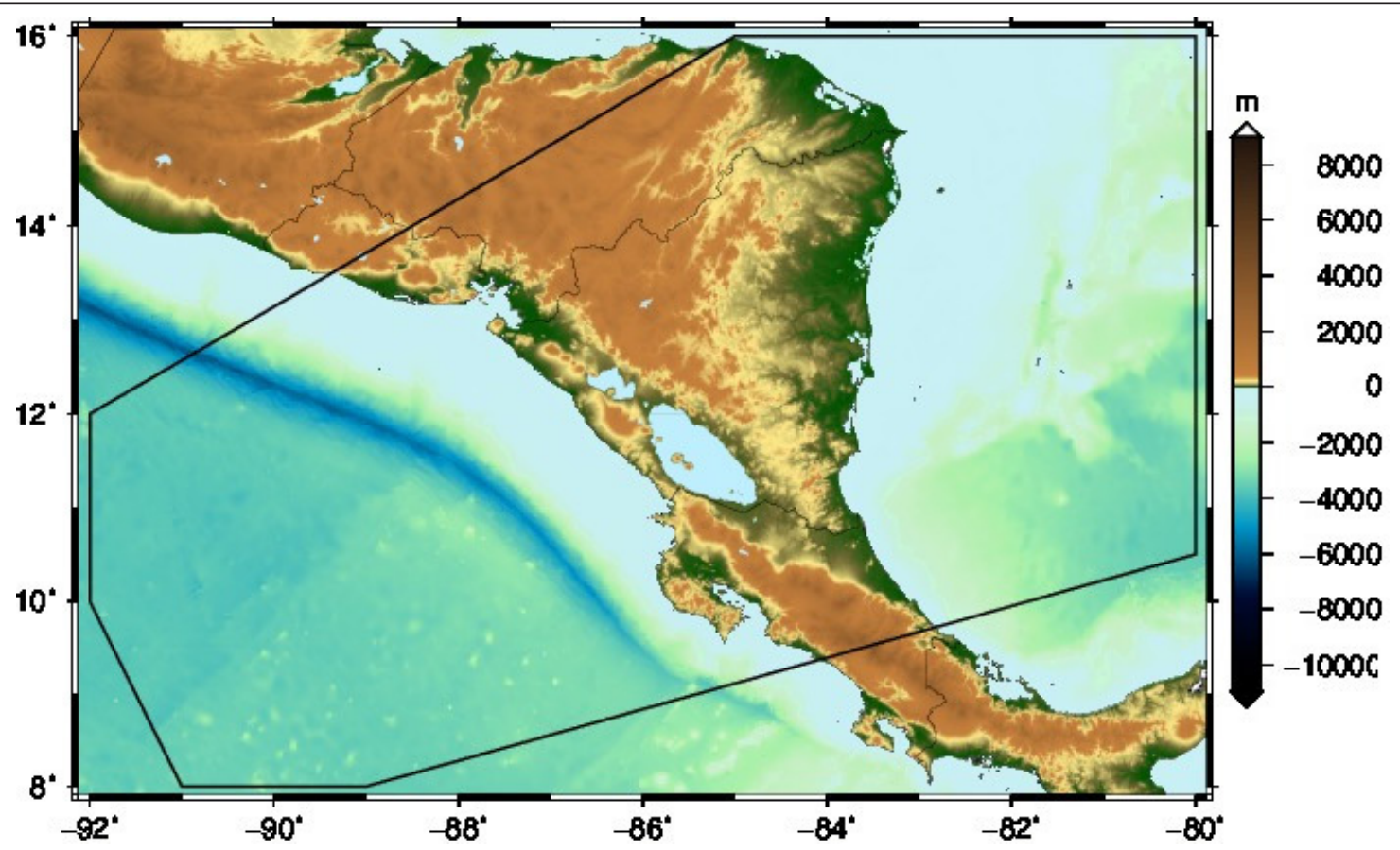

Figura 1. Contorno de selección para el catálogo confeccionado. Fuente: elaboración propia en base a gnuplot" (Williams y Kelley, 2017) y el GMT (Wessel y Smith, 1998).

se tradujo en un catálogo muy extenso, útil tanto para estudios detallados de sismicidad, como para estimados de peligrosidad sísmica. La región estudiada está limitada por la poligonal que se muestra en la figura 1. Esta área abarca toda Nicaragua (sus fronteras están marcadas por delgadas líneas negras en la figura), parte de los países vecinos y extensas zonas marítimas frente a ambas costas. Igualmente, se seleccionó un intervalo temporal desde 1520 hasta 2018. Este incluye desde los terremotos históricos hasta los últimos que para los que existe una mayor cantidad de información.

\section{Materiales y métodos}

\section{1) Catálogo de terremotos}

\section{1) Fuentes utilizadas}

Se disponía de dos elementos básicos: un catálogo preparado en el marco del proyecto RESIS-II (Benito et al., 2012; Molina et al., 2008) y el boletín de datos de la red de estaciones de Nicaragua, que comienza en 1975. Aparte de ello existen numerosas publicaciones que incluyen datos históricos, relocalizaciones de eventos, resultados de investigaciones temporales, etc. A esto se suman las compilaciones de datos que realizan agencias internacionales, las más importantes son las del ISC
(International Seismological Centre) y el USGS (United States Geological Survey). En detalle, las fuentes utilizadas son:

a) Catálogo del proyecto RESIS-II (Molina et al., 2008)

Catálogo en formato SEISAN (Otermöller et al., 2018)) compacto (1520-2007), donde la última columna de las magnitudes contiene una que denominan MW XXX, la cual corresponde a la seleccionada para su uso en amenaza sísmica, generalmente convertida a $\mathrm{M}_{\mathrm{w}}$ desde otra magnitud. Cada terremoto presenta sus coordenadas y tiempo de origen con indicación de la fuente de donde fueron tomadas.

\section{b) Catálogo de la red nicaragüense 1975/04 - 2018} (INETER, 2019)

Datos en formato SEISAN no compacto. Se caracteriza por la variabilidad en el registro: estable en los primeros años, disminución paulatina a principios de los 80 , y desaparición total desde 1983 hasta finales de 1992, en que se incrementa paulatinamente el nivel de detección. En 7/2017 se cambió el sistema de registro de SEISAN a SeiscomP3 (Behr et al., 2016). Se convirtieron los datos creados por el SeiscomP3 al formato SEISAN (Utheim \& Havskov, 2017), y se usaron estos para estimar $M_{w}$, que no es estimada en el SeiscomP3 en el último período analizado. 


\section{c) Relocalizaciones de eventos recientes}

Estudio de las réplicas de los terremotos de abril de 2014 en el volcán Momotombito y en la península de Chiltepe (Moreno et al., 2014) y de la serie de terremotos de octubre de 2015 en El Sauce (González et al., 2015), del terremoto de agosto de 2005 (French et al., 2010) del de Puerto Morazán en 2016 y de El Hoyo en 2016 (en un estudio de A. Cabrera) realizadas con el paquete de programas MJHD (Hurukawa \& Imoto, 1990, 1992).

\section{d) Catálogos preparados en ocasión del terremoto de Managua de 1972}

Catálogo de terremotos históricos (Leeds, 1974), réplicas del evento principal (Ward et al., 1973), relocalizaciones de eventos anteriores (Algermissen et al., 1974; Dewey \& Algermissen, 1974).

\section{e) Otras fuentes de datos locales}

Datos macrosísmicos de Nicaragua (Chuy, 1985).

\section{f) Compilaciones globales de los EE. UU. en línea}

Actual USGS: (1) EQH-PDE (1638-1995): combina los datos de terremotos históricos del "Earthquake History of the United States" y determinaciones instrumentales posteriores, (2) EHDF (1990 hasta 2013). (3) Selección en línea (USGS, 2019).

NOAA(National Oceanic and Atmospheric Administration): compilación de catálogos realizados por diferentes agencias, reunidos en unos CD (NOAA, 1996).

\section{g) Compilaciones globales del ISC}

ISC y su antecesor (ISS) (International Sesmological Summary): (1) boletines, hasta 1963 sin magnitudes, desde 1964 hasta la fecha, con magnitudes (2) en línea (ISC, 2020), donde se permiten dos variantes: ISS-ISC "completo", e ISS-ISC "revisado".

\section{h) Relocalizaciones de epicentros globales}

Con el algoritmo EHB como base (Engdahl et al.,1998), un catálogo de terremotos fuertes, denominado "Centennial" (Engdahl \& Villaseñor, 2002), accesible en el sitio web del USGS que abarca el período 1900-2007, y otro con umbrales más bajos de magnitud, para el período 1960 2017, accesible en el sitio web del ISC, bajo el nombre de catálogo EHB-ISC (Engdhal et al., 2020; ISC, 2020a;
Weston et al., 2018). Dentro del proyecto "ISC-GEM" (Bondár et al., 2015; Di Giacomo et al. 2018; Storchack et al., 2015) relocalización y ajuste de las magnitudes en diferentes ventanas energo(M)-temporales (Di Giacomo et al., 2015). Abarca desde 1900 hasta 2017 (El GEM corresponde al Global Earthquake Model, un proyecto internacional). Relocalización por el ISC de todos los eventos desde 1964 hasta la fecha (Storchak et al., 2017, 2020), usando el modelo de estructura de La Tierra "ak135" (Kennet et al., 1995; Montagner \& Kennet, 1995) y un algoritmo de cálculo mejorado (Bondár \& Storchak, 2011).

\section{i) Catálogo de centroides y tensores de momento}

Se da el valor del centroide, que no es igual al hipocentro habitual, que es considerada la mejor aproximación al foco puntual (Dziewonskii et al.,1981). Son muy confiables los valores de profundidad y magnitud. Existen desde 1961 hasta la fecha (GCMT, 2019).

\section{2) Selección de un formato}

Para comparar catálogos de terremotos es imprescindible que los mismos se encuentren expresados en el mismo formato, por lo que se seleccionó uno de trabajo. Se consideró como fundamental disponer del máximo posible de determinaciones de magnitud, así como de la precisión de las determinaciones hipocentrales y una cantidad "razonable" de información adicional. Se buscó considerar el máximo de elementos en el formato, excluyendo los de mecanismos focales, con referencia a que estos existen. Un elemento clave en este formato es código único que permitiera su enlace con otra información en un tratamiento de bases de datos relacionales. Para ello el mejor resultó el identificador de terremoto del ISC, un código de nueve números que no se repite, complementado por un número consecutivo negativo para aquellos terremotos no incluidos en el catálogo del ISC. Para realizar comparaciones entre diversos catálogos o entre registros de un mismo catálogo se incluyó un indicador formado por "día juliano y fracción", variable de doble precisión con siete cifras decimales, que permite una precisión de entre décima y centésima de segundo, mucho menos que el tiempo en que se puede diferenciar la ocurrencia de dos terremotos. El formato seleccionado, que denominaremos "CAT" se detalla en el Anexo 1 (materiales suplementarios).

La construcción de un catálogo completo requirió la consulta de todos los catálogos disponibles. Fue necesario confeccionar diversos programas de cómputo, los que en la etapa de preprocesamiento incluyen: 
Tabla 1

Orden de prioridad de los datos hipocentrales de las fuentes globales.

\begin{tabular}{ll}
\hline \multicolumn{1}{c}{ Orden } & \multicolumn{1}{c}{ Catálogos y boletines } \\
\hline 0 & $\begin{array}{l}\text { fuente que presenta los menores errores en la } \\
\text { localización }\end{array}$ \\
\hline 1 & catálogo EHB del ICTP \\
\hline 2 & catálogo ISC-GEM principal \\
\hline 3 & catálogo Centennial \\
\hline 5 & catálogo ISC-GEM suplementario \\
\hline
\end{tabular}

Fuente: elaboración propia.

Tabla 2

Orden de prioridad de los datos hipocentrales de las fuentes globales.

\begin{tabular}{ll}
\hline \multicolumn{1}{c}{ Orden } & \multicolumn{1}{c}{ Tipo de dato } \\
\hline 0 & $\begin{array}{l}\text { relocalizaciones realizadas en ocasión de terremotos } \\
\text { fuertes o series }\end{array}$ \\
\hline 1 & $\begin{array}{l}\text { fuente que presenta los menores errores en las } \\
\text { coordenadas }\end{array}$ \\
\hline 2 & determinaciones con gran número de estaciones \\
\hline 4 & $\begin{array}{l}\text { determinaciones por 4 o más estaciones (con error } \\
\text { medio cuadrático y errores por coordenadas) }\end{array}$ \\
\hline 5 & $\begin{array}{l}\text { determinaciones instrumentales con mayor } \\
\text { incertidumbre }\end{array}$ \\
\hline
\end{tabular}

Fuente: elaboración propia.

Tabla 3

Criterio de unión de datos de redes globales, red de Nicaragua y otras redes regionales.

\begin{tabular}{ll}
\hline \multicolumn{1}{c}{ Orden } & \multicolumn{1}{c}{ Criterio } \\
\hline 0 & $\begin{array}{l}\text { fuente que presenta los menores errores en las } \\
\text { coordenadas }\end{array}$ \\
\hline 1 & $\begin{array}{l}\text { para terremotos con determinaciones GEM o EHB } \\
\text { preferir estas sobre las redes locales }\end{array}$ \\
\hline 2 & $\begin{array}{l}\text { para terremotos con } \mathrm{mB}, \mathrm{MS} \geq 4,5 \text { preferir } \\
\text { localizaciones del ISC o del GS-NEIS sobre las } \\
\text { redes locales, si no indican errores }\end{array}$ \\
\hline 3 & $\begin{array}{l}\text { para terremotos con mB, MS<4,5 preferir la red de } \\
\text { Nicaragua, cuando indique los errores }\end{array}$ \\
\hline 5 & $\begin{array}{l}\text { preferir la red de Nicaragua sobre otras redes } \\
\text { regionales }\end{array}$ \\
\hline
\end{tabular}

Fuente: elaboración propia.
- conversión de formatos iniciales al formato CAT;

- unión de catálogos, selección de partes (tiempo, coordenadas, magnitud);

- intercalación de dos catálogos;

- $\quad$ análisis de presencia de tipos de magnitud en un catálogo (eliminación de duplicados).

La parte más compleja del trabajo es la comparación entre catálogos y selección de uno resultante. Se confeccionaron programas interactivos para tomar decisiones caso a caso. Primero se confeccionó un catálogo que contenía varios registros por terremoto, resultado de la unión de diferentes fuentes, entre las que se seleccionaba la que se consideraba de mayor calidad. Para estimar esa "calidad" en primer lugar se usaron los indicadores de esta que proporcionaban sus autores, y se estableció un orden de selección entre fuentes en dependencia del conocimiento sobre el método de cálculo utilizado. Con preferencia se tomaron las relocalizaciones, seguidas por las localizaciones efectuadas usando un gran número de estaciones. Esto es válido tanto para redes locales como globales. En el caso de los terremotos más fuertes predominarán los resultados de las redes globales, aunque pueden ser mejores los estimados de profundidad de las locales. El orden de prioridad en las fuentes globales establecido se muestra en la tabla 1. Los criterios usados para esta selección están en las publicaciones citadas para cada caso. Nótese que esto es para las coordenadas. Para las magnitudes se trató de considerarlas todas, y el orden de prioridad entre ellas solo se consideró a la hora de preparar una versión del catálogo unificada a $\mathrm{M}_{\mathrm{W}}$.

Para el caso de las fuentes locales el orden de prioridad se indica en la tabla 2.

Los criterios de prioridad al combinar ambos tipos de datos se muestran en la tabla 3 .

\section{3) Magnitudes presentes en el catálogo y sus fuentes}

Un problema clave en cualquier catálogo de terremotos refiere a las fuentes desde donde se extraen los datos que en él se incluyen. Si bien para fecha y coordenadas se hizo una selección de fuente de acuerdo a su confiabilidad, en el caso de las magnitudes se decidió poner todas las que se han determinado para cada terremoto hasta el límite de las 12 magnitudes posibles en el formato seleccionado. Solo en contados casos, en el proceso de confección del catálogo fue necesario eliminar alguna determinación de magnitud por exceder dicho límite. 
Los tipos de magnitud presentes son, fundamentalmente: $\mathrm{m}_{\mathrm{B}}, \mathrm{M}_{\mathrm{S}}, \mathrm{M}_{\mathrm{W}}, \mathrm{M}_{\mathrm{C}}\left(\mathrm{o} \mathrm{M}_{\mathrm{D}}\right)$ y $\mathrm{M}_{\mathrm{L}}$. La $\mathrm{m}_{\mathrm{B}}$ en su inmensa mayoría corresponde a determinaciones con ondas de corto período, las llamadas $\mathrm{m}_{\mathrm{b}} \mathrm{y} \mathrm{m}_{\mathrm{pv}}$. La $\mathrm{M}_{\mathrm{C}}$, es la magnitud por coda, un tipo de magnitud por duración (Bormann, 2002). Puede haber otros tipos de magnitud, pero en menor cuantía dentro del catálogo. Entre ellas debe destacarse la $M_{B}$ determinada con registros de período medio, que está apareciendo en el catálogo de la red nicaragüense desde que se introdujo el sistema SeiscomP3. Para evitar confusiones, esas magnitudes se renombraron a MX.

Las fuentes de las magnitudes son variadas. Las más confiables de las $m_{B}$ y $M_{S}$ son del ISC y de la red global operada por los EE. UU., que aparecen con diferentes claves de agencia y fueron renombradas a NEIC (National Earthquake Information Centre, otra de las denominaciones con que aparecen las determinaciones de los EE. UU.). Otras determinaciones muy confiables son la $\mathrm{M}_{\mathrm{W}}$ del proyecto CMT (Centroid Moment Tensor) de la Universidad de Harvard (clave de agencia HRV, o más reciente GCMT), la del EHB del ISC. El GEM presenta solo magnitudes $\mathrm{M}_{\mathrm{w}}$, pero en muchos casos son convertidas de otras magnitudes (la clave de estación en el catálogo lleva al final 2 caracteres $\mathrm{Qs}, \mathrm{Q}=\mathrm{A} / \mathrm{B} / \mathrm{C} \rightarrow$ calidad, $\mathrm{s}=\mathrm{p} / \mathrm{d} \rightarrow$ $\mathrm{p}=$ proxy[convertida], $\mathrm{d}=$ directa). $\mathrm{La} \mathrm{M}_{\mathrm{W}}$ aparece en los últimos tiempos también en las redes locales.

En el catálogo aparecen también muchas magnitudes de diferente tipo de la agencia 'IDC' que son menos confiables y se obvian, de existir otras, en los análisis posteriores.

Un caso especial es el de las magnitudes presentes en el catálogo del proyecto RESIS-II (Molina et al., 2008). El formato utilizado permite tres magnitudes, pero en muchos casos solo aparece la $\mathrm{M}_{\mathrm{w}}$, sin indicar la fuente a partir de donde fue convertida. En los casos en que existía algún otro valor de magnitud, no se tomó el de MW XXX (forma de representarla en ese catálogo). Esto se hizo en el proceso de unificación de los distintos catálogos básicos, pero hay otros eventos que no fueron objeto de la comparación automática que aún tienen esa MW XXX adicional, la cual debe ser eliminada en futuras revisiones, si aparecen los datos iniciales. El origen de estas MW XXX aisladas es tratado por Benito \& Torres (2009), dejando claro el origen de la mayoría de ellas. Otra cuestión que aún no ha podido ser aclarada es el origen de determinaciones $\mathrm{M}_{\mathrm{S}}$ y $\mathrm{M}_{\mathrm{W}}$ con agencia "CAL". Hay bastantes en el catálogo y no se sabe cuan confiables son.

\section{2) Transformación del catálogo en uno con magnitudes $M_{w}$}

Para realizar análisis estadísticos de un catálogo es necesario que todas las magnitudes se expresen en una sola escala, lo que denominaremos "catálogo unificado". El catálogo que tiene un máximo de 12 magnitudes diferentes debe ser convertido a uno donde aparezca un solo tipo de magnitud, en este caso $\mathrm{M}_{\mathrm{W}}$. Para ello, todas las magnitudes deben ser convertidas a $\mathrm{M}_{\mathrm{W}}$, por lo que hay que establecer las relaciones entre ellas. Esta magnitud se determina tanto por agencias globales como por redes locales, siendo más estable la determinada por las primeras; entre ellas, las más confiables son NEIC, ISC, GEM y HRV-GCMT. Se usa un promedio de esas magnitudes en cada terremoto al que se le llama $\mathrm{M}_{\mathrm{W}}$ (media) como base para el catálogo final. En el Anexo 2 (materiales suplementarios) se referencian todas las agencias o redes cuyos datos se utilizaron.

Se realizaron trabajos de regresión lineal entre dos tipos de magnitud. Las regresiones son del tipo:

$$
M_{2}(\text { final })=a+b \cdot M_{1}(\text { inicial })(1)
$$

donde se determinan parámetros $\left(\mathrm{a}, \sigma_{\mathrm{a}}, \mathrm{b}, \sigma_{\mathrm{b}}\right)$, así como el coeficiente de correlación " $r$ ". Como no siempre aparecen poblaciones de tipo $\left[\mathrm{M}_{\mathrm{i}}, \mathrm{M}_{\mathrm{W}}\right]$ a veces no queda más remedio que buscar una expresión formada por una conversión doble $\mathrm{M}_{\mathrm{i}} \rightarrow \mathrm{M}_{\mathrm{T}} \rightarrow \mathrm{M}_{\mathrm{W}},\left(\mathrm{M}_{\mathrm{T}}\right.$ es una magnitud de tránsito), menos confiable que la conversión directa. Esta combinación de regresiones conduce a una expresión del tipo:

$$
M_{W}(\text { media })=c+d \cdot M_{i}
$$

donde $\mathrm{c}=\mathrm{b}_{2} \cdot \mathrm{a}_{1}+\mathrm{a}_{2}, \mathrm{~d}=\mathrm{b}_{2} \cdot \mathrm{b}_{1}$, el subíndice " 1 " corresponde a la regresión $\left(\mathrm{M}_{\mathrm{i}}, \mathrm{M}_{\mathrm{T}}\right)$ y el " 2 " a la $\left(\mathrm{M}_{\mathrm{T}}, \mathrm{M}_{\mathrm{W}}\right)$.

La selección de las muestras $\left(\mathrm{M}_{1}, \mathrm{M}_{2}\right)$ a usar para el análisis de regresión se hizo de forma automática usando programas en FORTRAN y scripts en PostgreSQL sobre bases de datos. El proceso es, a partir del catálogo inicial, preparar un catálogo auxiliar que llene sus 12 posiciones de magnitud con las que se quiera analizar, importar ese catálogo a una base de datos, y finalmente realizar en esta la selección de las muestras $\left(\mathrm{M}_{1}, \mathrm{M}_{2}\right)$ a analizar. Para las magnitudes $\mathrm{m}_{\mathrm{b}} \mathrm{y} \mathrm{M}_{\mathrm{S}}$, las más comunes en los catálogos sismológicos, Scordilis (2006) determinó unas fórmulas de conversión $\mathrm{M}_{\mathrm{W}}\left(\mathrm{m}_{\mathrm{B}}, \mathrm{M}_{\mathrm{S}}\right)$ de validez global y un alto nivel de confiabilidad, que fueron tomadas como válidas en este trabajo y fueron utilizadas como magnitudes de tránsito $\left[\mathrm{m}_{\mathrm{B}}\right.$ (media) y $\mathrm{M}_{\mathrm{S}}$ (media) $]$. 


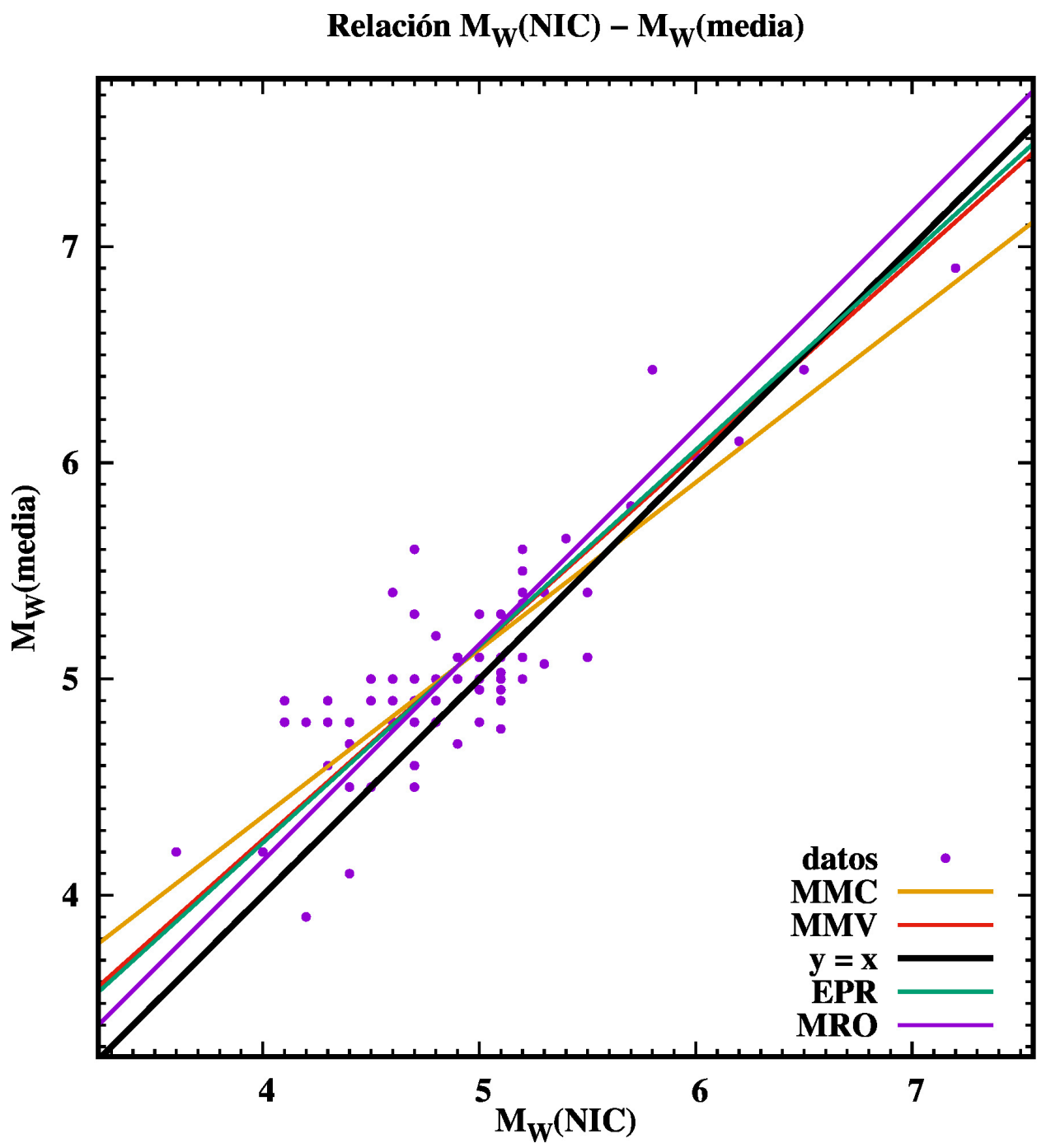

Figura 2. Ejemplo de análisis de regresión. Fuente: elaboración propia en base a gnuplot" (Williams y Kelley, 2017) y el GMT (Wessel y Smith, 1998).

Un caso importante es el de las magnitudes por longitud de registro determinadas en los trabajos de campo por el terremoto de Managua de 1972 (Ward et al., 1973). De un total de 170 terremotos, hay 168 que solo tienen ese tipo de magnitud obtenida por la fórmula de Lee et al. (1972) por duración de coda. Para magnitudes determinadas por esa fórmula, Bakun (1984) determinó una conversión a $\mathrm{M}_{0}$ :

$$
\lg \left(M_{0}\right)=1,2 \cdot M_{D}+1,7
$$

Esto permite hacer una transformación doble $\mathrm{M}_{\mathrm{C}} \rightarrow \mathrm{M}_{0}$ $\rightarrow \mathrm{M}_{\mathrm{W}}$, usando la fórmula de conversión $\mathrm{M}_{0} \rightarrow \mathrm{M}_{\mathrm{w}} \mathrm{de}$ Hanks \& Kanamori (1979):

$$
\lg \left(M_{0}\right)=1,5 \cdot M_{W}+16,1
$$

Se probaron cuatro métodos de ajuste (mínimos cuadrados (MMC), máxima verosimilitud (MMV), regresión ortogonal (MRO) y eje principal reducido (EPR), decidiéndose dar preferencia al segundo. En la figura 2 se presenta la conversión de $\mathrm{M}_{\mathrm{W}}$ (NIC) a $\mathrm{M}_{\mathrm{W}}$ (media).

El centro CASC-CADCG reporta $\mathrm{M}_{\mathrm{D}}$ y se comprobó que no hubo cambios en su determinación por la cercanía de las relaciones $\mathrm{M}_{1}=\left[\mathrm{M}_{\mathrm{D}}(\mathrm{CASC}), \mathrm{M}_{\mathrm{D}}(\mathrm{CADCG})\right]$ con $\mathrm{m}_{\mathrm{B}}$ (media) y $\mathrm{M}_{\mathrm{S}}$ (media) y se unieron en $\mathrm{M}_{\mathrm{D}}(\mathrm{CAC} 2)$. A más del $90 \%$ de los terremotos pudo aplicársele una transformación de magnitud directa. Se usó la fórmula (2) preferentemente si el tránsito se podía hacer a través de las fórmulas de Scordilis (2006). Debe recordarse que este tipo de conversión es menos precisa. Los parámetros de 
las regresiones directas $\mathrm{M}_{1} \rightarrow \mathrm{M}_{2}$ se presentan en la tabla 1 del Anexo 3 (materiales suplementarios).

3) Metodología de análisis de eventos relacionados

Es conocido que los terremotos fuertes son seguidos en general por numerosas réplicas, lo que eleva el nivel medio de ocurrencia de eventos. Se estudian estos casos con dos objetivos, uno específico de análisis del proceso terremoto-principal-réplicas, y otro de preparar un catálogo que no contenga eventos relacionados (se usan las denominaciones "limpio" y declustered). Por tanto, los procesos premonitores - terremoto principal - réplicas, así como las series de terremotos se consideran como eventos simples, con una magnitud determinada a partir de la energía liberada en el proceso, que acostumbra a denominarse "magnitud equivalente".

Existen diversos algoritmos que realizan este proceso, y entre ellos fue seleccionado el desarrollado por Reasenberg (1985), para el cual está accesible el programa cluster2000x (Reasenberg, 2000). Dicho programa fue modificado para que procesara eventos en formato CAT y se cambió la fórmula para calcular el momento sísmico equivalente, de la original (una $\mathrm{M}_{\mathrm{CODA}}$ del USGS) a otra para $\mathrm{M}_{\mathrm{W}}$ (Hanks \& Kanamori, 1979), amén de otros cambios menores.

El método busca la construcción de una ventana espacio temporal $[\tau(M), \Delta(M)]$ en la que se encuentran los eventos relacionados, donde " $\tau$ " se calcula como:

$$
\tau=[-\lg (1-P)]^{t} / 10^{[2 / 3 \cdot(\Delta M-1)]}
$$

donde $\Delta M=M_{p r i}-M_{\min }\left(M_{p r i}\right.$ - magnitud del terremoto principal, $M_{\min }$ depende de las observaciones), $\mathrm{P}$ es la probabilidad de que en un tiempo t (días) ocurra una réplica (se fija en el programa en 0,99 ); $\tau$ viene a ser el tiempo entre dos eventos sucesivos.

“ $\Delta$ ", denominada distancia de interacción viene dada por:

$\Delta=r_{\text {fact }} \cdot a(M, \Delta \sigma), \quad \lg (a)=0,4 \cdot M_{\text {pri }}-(\lg (\Delta \sigma)) / 3-1,45$

donde $a(M, \Delta \sigma)$ es el radio de una falla circular (Kanamori $\&$ Anderson, 1975) correspondiente a un terremoto de magnitud $\mathrm{M}$ y una caída de esfuerzos (stress drop) $\Delta \sigma$. Para $\Delta \sigma=30$ bars (fijado en el programa), los dos últimos términos de la fórmula para $\lg (a)$ equivalen a 0,11 . El resultado de este programa es un nuevo catálogo, sin los eventos relacionados, con el mismo formato que el original, solo que en la posición 12 de las magnitudes para el terremoto principal se pone el valor de magnitud equivalente del grupo de eventos relacionados (que denominan cluster), y para los eventos no relacionados se copia la $M_{W}$ de la primera posición. La "magnitud equivalente" se calcula transformando las magnitudes individuales a energía, sumando estas y convirtiendo la suma a magnitud nuevamente. Se pueden obtener catálogos individuales para agrupamientos seleccionados.

El comportamiento temporal de los agrupamientos de terremotos ha sido estudiado por diferentes autores y su modelación se remonta a finales del siglo sXIx (Omori, 1894). De ahí parte la llamada "ley de Omori”" (Utsu, 1961):

$$
n(t)=K \cdot(t+c)^{-p}
$$

donde $n(t)$ es el número de eventos en la unidad de tiempo $(t)$, generalmente días, y $(K, c, p)$ son constantes a determinar. Esta fórmula es llamada "modificada de Omori". Hay veces que un proceso de este tipo presenta una clara diferenciación en secuencias que siguen a alguno de los terremotos más fuertes, por lo que existe una modificación de la fórmula anterior

$n(t)=K_{1} \cdot\left(t+c_{1}\right)^{-p_{1}}+H\left(t-T_{2}\right) \cdot K_{2} \cdot\left(t+c_{2}\right)^{-p_{2}}+H\left(t-T_{3}\right) \cdot K_{3} \cdot\left(t+c_{3}\right)^{-p_{3}}$

donde $H($.$) es la función de Heaviside. Los términos$ en (2) y (3) corresponden al caso de 2 y 3 secuencias respectivamente, desapareciendo el ultimo miembro de la ecuación si solo hay 2 secuencias. Aquí se multiplica el número de constantes a determinar, con 3 por cada secuencia. El número acumulativo de terremotos se obtiene en ambos casos como:

$$
N_{a c}(t)=\int_{0}^{t} n(\tau) \cdot d \tau
$$

Un análisis de este tipo de fórmulas puede verse en (Utsu et al., 1995) y (Ogata, 1999).

Otro modelo muy usado es el ETAS (Epidemic Type Aftershock Sequence), que se basa en la teoría de los procesos puntuales Ogata (1988), e incorpora el modelo de Omori. La tasa de ocurrencia de réplicas un tiempo " $t$ " después de que ocurrió el i-ésimo terremoto es:

$n_{i}(t)=K \cdot \exp \left[\alpha \cdot\left(M_{i}-M_{z}\right)\right] /\left(t-t_{i}+c\right)^{p}, t>t_{i}$

donde las constantes $(K, \alpha, c, p)$ son comunes para toda la región y $M_{z}$ es la magnitud umbral. La tasa de ocurrencia total para toda la serie es: 


$$
\lambda(t)=\mu+\sum n_{i}(t)
$$

donde la suma se hace para todas las " $i$ " que satisfagan $t_{i}<t$. El número acumulativo de eventos $N_{a c}(t)$ en el tiempo ' $t$ ' desde $t_{0}$ viene dado por:

$N_{a c}(t)=\mu\left(t-t_{0}\right)+K \cdot \sum \exp \left[\alpha \cdot\left(M_{i}-M_{z}\right)\right] \cdot\left[c^{1-p}-\left(t-t_{i}+c\right)^{1-p}\right] /(p-1)$

Otro aspecto para tomar en consideración es el cálculo de la caída de esfuerzos efectiva total de cada agrupamiento [effective stress drop - Fischer \& Hainzl (2017)]. Para ello se considera la fórmula desarrollada por esos autores:

$$
\Delta \tau_{\text {efec }}=\frac{7}{16} \cdot \frac{\sum M_{0}}{R^{3}}
$$

donde la suma se realiza sobre todos los eventos de la serie y el valor de $\mathrm{R}$ es la dimensión de la falla activada. El valor de $\mathrm{M}_{0}$ se calcula a partir de las magnitudes $\mathrm{M}_{\mathrm{w}}$ de los terremotos por la fórmula (4). El valor de R se calcula considerando una falla circular de área equivalente al área real ocupada por el agrupamiento. Para ello se traza el contorno que engloba a los eventos con el programa "ConcaveHull.py" (Moreira \& Santos, 2007) y se calcula su área interior usando el algoritmo de Rokne (1995).

\section{5) Metodología de análisis la sismicidad}

La forma más común de realizar una caracterización de la sismicidad es a través de la construcción de los gráficos magnitud-frecuencia (M-F) y la estimación de sus parámetros fundamentales $\left(\mathrm{a}, \mathrm{b}, \mathrm{M}_{\max }\right.$ ). Existen diferentes formas de representar estos gráficos y un análisis de muchas de ellas se puede encontrar en (Utsu, 1999). En este caso se usa una forma particular de representar estos gráficos (Alvarez et al., 2017), que tiende a buscar expresiones analíticas de las cantidades usadas para determinar sus parámetros. La forma general de la ley magnitud-frecuencia es:

$n(M)=10^{a-b \cdot M}, \quad \lg [n(M)]=a-b \cdot M, \quad M<M_{\max }(14)$

donde $n(M) \cdot d M$ - cantidad de terremotos en el intervalo e/ $M$ y $M+d M$ y en el intervalo de tiempo $t$. Aquí la magnitud $n(M)$ es continua y representa la densidad de la frecuencia de ocurrencia de los terremotos, generalización matemática muy cómoda para el desarrollo posterior de la teoría. Los gráficos magnitud frecuencia "distributivos" se confeccionan a partir del número de terremotos en intervalos de ancho $\triangle M$. En otras palabras, se determinan los valores $\left(N_{i}, M_{i}\right)$, donde $N_{i}$-número de terremotos dentro del intervalo de longitud $\Delta M$, con valor central
$M_{i}$, normalizado en el tiempo. Se simbolizan por $N\left(M_{i}\right)$, y corresponden a $N\left(M_{i}-\Delta M / 2, M_{i}+\Delta M / 2\right)$. Su expresión analítica es:

$N\left(M_{i}\right)=10^{a-b \cdot M_{i}} \cdot\left[10^{b \cdot \Delta M / 2}-10^{-b \cdot \Delta M / 2}\right] /(b \cdot \ln 10)=10^{a-b \cdot M_{i}} \cdot F(b, \Delta M)$

Se puede demostrar que para pequeños valores de $\Delta M$ $F(b, \Delta M) \approx \Delta M$, lo que permite obtener expresiones aproximadas, y de forma recursiva ir aumentando la precisión. El llamado gráfico acumulativo corresponde al caso en que se calcula el número de terremotos en el intervalo (M- $\delta$, Mmax), donde $\delta=\Delta \mathrm{M} / 2$.

$$
N_{\Sigma}(M)=\frac{n(M)}{b \cdot \ln 10} \cdot 10^{b \cdot \delta} \cdot\left\{1-10^{-b \cdot\left(M_{\max }-M+\delta\right)}\right\}
$$

En el caso práctico, en la división en intervalos, el último puede tener una longitud diferente que los anteriores para ocupar todo el rango de existencia de la magnitud.

Los parámetros $(a, b)$ se calculan por regresión a partir de los gráficos experimentales $\left[\mathrm{N}\left(\mathrm{M}_{\mathrm{i}}\right), \mathrm{M}_{\mathrm{i}}\right]$, pero el valor de $M_{\max }$ es mucho más difícil de determinar. Se pueden usar diversos valores: máximos reportados, valores tomados de otras zonas similares desde el punto de vista sismotectónico, estimados por dimensiones de fallas o por métodos estadísticos. Entre los métodos estadísticos destaca el propuesto por Kijko (2004).

Se tiene una serie de " $n$ " magnitudes observadas en el intervalo $\left[m_{\text {min }}, M_{\text {max }}\right]$. Se asume que están ordenadas en orden ascendente $m_{1} \leq m_{2} \leq \ldots \leq m_{n}$. Observemos que $m_{n}$, que es la mayor magnitud observada (que se indica como $M_{\max }^{o b s}$ ) tiene la FD

$$
F_{M_{n}}(m)=\left\{\begin{array}{cc}
0 & m<m_{\min } \\
{\left[F_{M}(m)\right]^{n}} & m_{\min } \leq m \leq M_{\max } \\
1 & m>M_{\max }
\end{array}\right.
$$

Teniendo en cuenta que el valor de la mayor magnitud observada, $M_{\text {max }}^{o b s}$, es el mejor estimado insesgado de $E\left[M_{n}\right]$, luego de un proceso más o menos largo se obtiene para $M_{\max }$ :

$$
\begin{gathered}
M_{\max }=M_{\text {max }}^{o b s}+\int_{m_{\min }}^{M_{\max }}\left[F_{M}(m)\right]^{n} \cdot d m \\
\Delta=\int_{m_{\min }}^{M_{\max }}\left[F_{M}(m)\right]^{n} \cdot d m, \operatorname{Var}\left(\widehat{M}_{\max }\right)=\sigma_{M}^{2}+\Delta^{2}
\end{gathered}
$$

donde $\sigma_{\mathrm{M}}^{2}$ es el error estándar en la determinación de $M_{\max }^{o b s}$ 
La estimación de la $\mathrm{M}_{\max }$ se hace por métodos iterativos complejos que son discutidos en su mayoría en (Kijko \& Sing, 2011). Además de eso, Kijko preparó un programa sobre Matlab que realiza el trabajo (Kijko, 2020) permitiendo elegir el método iterativo a aplicar.

Se complemente el análisis de la sismicidad con gráficos de liberación de energía y espacio-temporales. Para realizar análisis de energía liberada se usa la fórmula:

$$
\lg E[\operatorname{erg}]=11,8+1,5 \cdot M_{S}
$$

Debe señalarse que se introduce un ligero error con el uso de esta fórmula si se evalúa en $\mathrm{M}_{\mathrm{w}}$, pero como es uniforme para todo el catálogo, no influye en los métodos de análisis que se van a emplear. En los gráfico espacio-temporales cada terremoto se representa por una barra horizontal, de dimensión proporcional a la longitud de falla determinado a partir de la fórmula de Wells \& Coppersmith (1994):

$\lg (L)=-2,44+0,59 \cdot M_{w} \Rightarrow L(M)=0,0006 \cdot \exp \left(1,58878 \cdot M_{w}\right)$

\section{Resultados}

\section{1) Catálogo de terremotos}

Este proceso de formación del catálogo fue largo. Se procesaban grupos de catálogos antes de unirlos con otros, hasta que finalmente se llegó a uno final. Los programas confeccionados permitían ir eliminando duplicados paso a paso; se usaban repetidas veces cambiando la distancia y el tiempo para la comparación de dos registros consecutivos. En otras ocasiones se buscaban grupos de eventos cercanos y se actualizaba a mano la selección. Luego de múltiples revisiones se llegó a la versión con un catálogo con un solo registro por terremoto. La inmensa mayoría de los eventos con registros duplicados pudo ser analizada de forma semiautomática, y se encontraba dentro de umbrales $\delta \mathrm{t}_{0}=20 \mathrm{seg}$ y $\delta \Delta=40 \mathrm{~km}$. Sin embargo, un número no despreciable se encontraba muy alejado espacialmente. Incluso en muchos casos de terremotos fuertes la diferencia entre el USGS y el ISC podía alcanzar $\delta \Delta>100 \mathrm{~km}$. Las localizaciones del ISC eran más cercanas a las de la red nicaragüense que las del USGS. La diferencia resulta muy grande también entre las diferentes redes de países centroamericanos. Se prefirió siempre en este último caso la de la red nicaragüense, a no ser que los errores reportados fueran excesivos. El ISC añade localizaciones de eventos por redes con localizaciones poco confiables como PMEL e IDC. Se procuró eliminarlas siempre y cuando existiese alguna localización relativamente cercana de alguna de las redes más confiables. En el proceso se unieron registros de eventos que en algunos casos podrían tener diferencias $\delta \Delta>200 \mathrm{~km}$; esto se hizo luego de valorar la cercanía en tiempo y la presencia de magnitudes comparables entre las fuentes.

La inmensa mayoría de los eventos tiene coordenadas y tiempo de origen determinados por la red nicaragüense (1975-2017), y gran parte de ellos tienen estimaciones de los errores. Sin embargo, no se está completamente seguro de esas localizaciones hipocentrales; los errores pueden alcanzar valores muy altos y siempre está presente el error sistemático inherente al modelo de corteza utilizado. En su última versión, el catálogo consta de 76.935 terremotos, de los cuales 74.500 tienen al menos una determinación de magnitud. Se adjunta en los "materiales suplementarios".

El nuevo catálogo con magnitudes transformadas (materiales suplementarios) usa las cuatro primeras posiciones para magnitud (Anexo 1) para $M_{W}, M_{L}$ (no convertida), $M_{C}, M_{D}$ (no convertida) y $\mathrm{M}_{\mathrm{O}}$ (otro tipo, no convertida). Las magnitudes $\mathrm{M}_{\mathrm{W}}$ presentes tienen una clave que indica el proceso de su conversión (tabla 2, Anexo 3 de materiales suplementarios). Solo para 24 terremotos no se pudo convertir la magnitud a $\mathrm{M}_{\mathrm{W}}$. De tal forma se obtiene un catálogo unificado en $\mathrm{M}_{\mathrm{W}}$ (materiales suplementarios). Debe señalarse que la conversión de magnitudes no es un proceso recomendable para el estudio de terremotos individuales. Se hace en los catálogos para su procesamiento estadístico, lo que compensa variaciones puntuales.

Para visualizar aproximadamente el contenido del catálogo se prepararon varios mapas de epicentros (figura 3):

(a) Etapa histórica; (b) registro instrumental, procesamiento manual; (c) predominio de redes regionales; (d) etapa actual. Se ve que en la etapa histórica la mayoría de los eventos están en la zona continental y casi no hay eventos profundos. La situación cambia con el registro instrumental, pero con la determinación manual de hipocentros la dispersión es bastante grande. En la etapa de predominio de redes regionales se perfila mejor el patrón de la sismicidad, y el umbral de detección puede bajar por períodos y zonas. Finalmente, la etapa actual se caracteriza por redes locales más densas, cual es el caso Nicaragua, aumentando la precisión de los hipocentros y disminuyendo el umbral de detección.

\section{2) Análisis de la sismicidad a partir el catálogo}

Tras una corrida del programa "cluster2000x" (usando los valores de $r_{\text {fact }}(10)$ y $\tau_{\max }(1440 \mathrm{~min})$ implícitos, una $M_{\text {min }}$ de 2,0 e ignorando los errores en coordenadas) sobre 


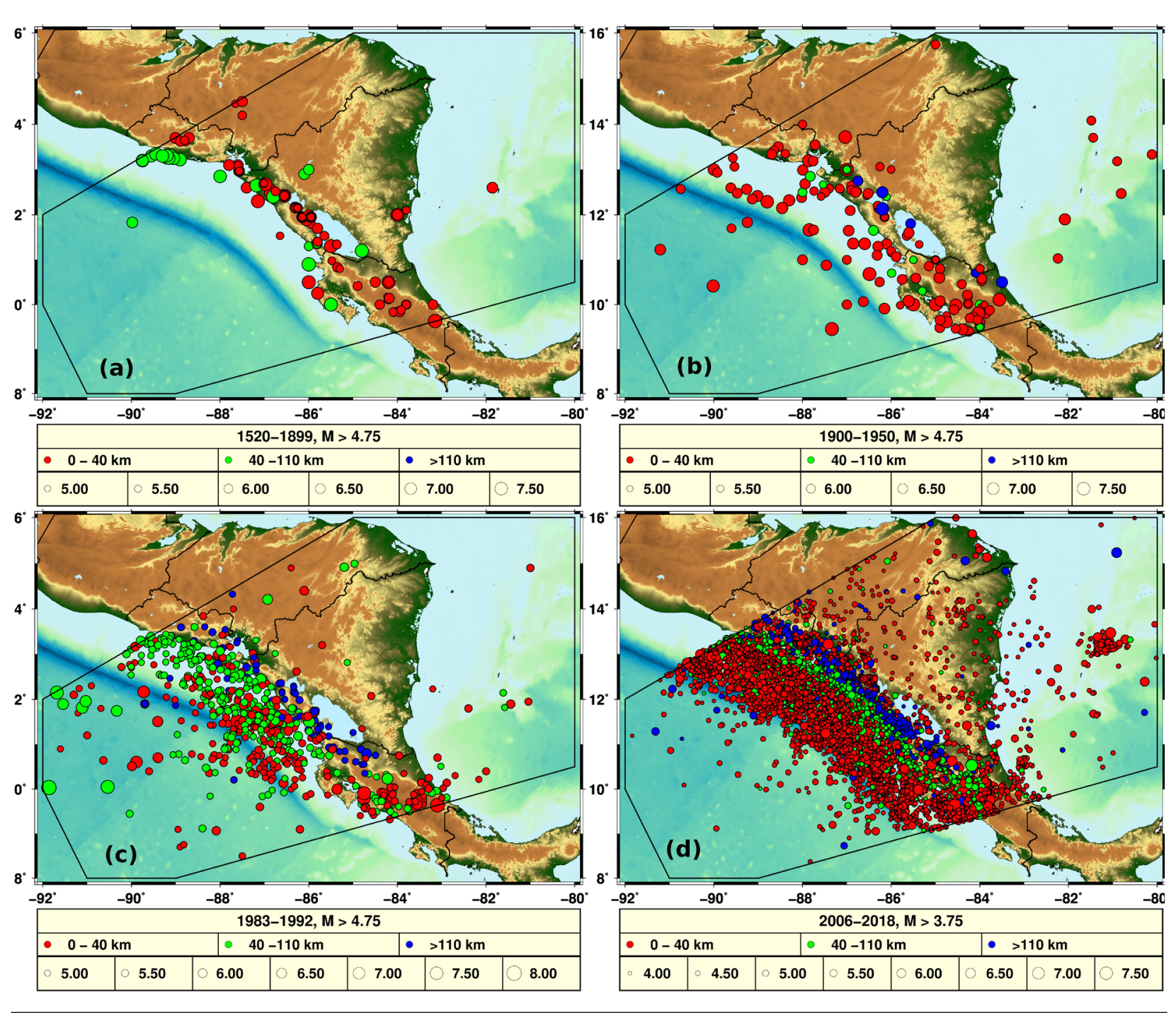

Figura 3. Mapas de epicentros. Fuente: elaboración propia en base a gnuplot" (Williams y Kelley, 2017) y el GMT (Wessel y Smith, 1998).

el catálogo unificado en $\mathrm{M}_{\mathrm{w}}$, se encontró que 11.528 estaban agrupados en 1.132 grupos. La cantidad de eventos en un grupo puede variar desde dos en adelante, por lo que no para todos los grupos se puede realizar un análisis estadístico. Igualmente se obtuvo, y se obtuvo un catálogo "limpio" con 65.080 terremotos sin eventos relacionados. Debe señalarse que de variar los parámetros $\mathrm{r}_{\text {fact }}, \tau_{\max } \mathrm{y} \mathrm{M}_{\text {min }}$, se pueden obtener un catálogo "limpio" y un número de grupos diferentes, así como un número diferente de terremotos en cada grupo.

\section{1) Estudio de grupos seleccionados}

Para realizar el estudio de procesos premonitoresterremoto principal-réplicas o de series es imprescindible que los grupos sean bastante grandes. Se seleccionaron ocho procesos que contenían más de 400 eventos cada uno. Todos ellos corresponden a la depresión de Nicaragua. Aunque existen procesos de este tipo en la zona de subducción, el número de eventos de cada proceso es insuficiente, ya que el umbral de detección de magnitudes es superior al de los eventos de la depresión. Los procesos seleccionados se muestran en la tabla 4. En ella aparece una clasificación del tipo de proceso en premonitoresterremoto principal-réplicas (PTR) y series (S), aunque debe señalarse que en general los premonitores, o no existen, o son muy escasos.

Estos agrupamientos de terremotos han sido estudiados por diferentes autores, destacándose el trabajo de Segura 
Tabla 4

Agrupamientos de terremotos en la depresión de Nicaragua.

\begin{tabular}{llc}
\hline \multicolumn{1}{c}{ Descripción } & Tipo & \# eventos \\
\hline $\begin{array}{l}\text { agosto de 1999, volcán Cerro Negro } \\
\text { junio-agosto de 2000, laguna de Apoyo, Departamento de } \\
\text { Masaya }\end{array}$ & PTR & 591 \\
\hline $\begin{array}{l}\text { enero 2001, península de Chiltepe, lago Xolotlán } \\
\text { agosto-septiembre 2005, isla de Ometepe, lago Cocibolca }\end{array}$ & S o PTR & 417 \\
\hline $\begin{array}{l}\text { abril-julio 2014 mitad occidental del lago Xolotlán } \\
\text { septiembre-noviembre 2015, El Sauce, Departamento de } \\
\text { León }\end{array}$ & PTR & 423 \\
\hline $\begin{array}{l}\text { junio-agosto de 2016, Puerto Morazán, Departamento de } \\
\text { Chinandega }\end{array}$ & PTR & 620 \\
\hline \begin{tabular}{l} 
septiembre-octubre de 2016, volcán El Hoyo \\
\hline
\end{tabular} & S & 926 \\
\hline
\end{tabular}

PTR - premonitores-terremoto principal-réplicas, S-serie.

Fuente: elaboración propia.

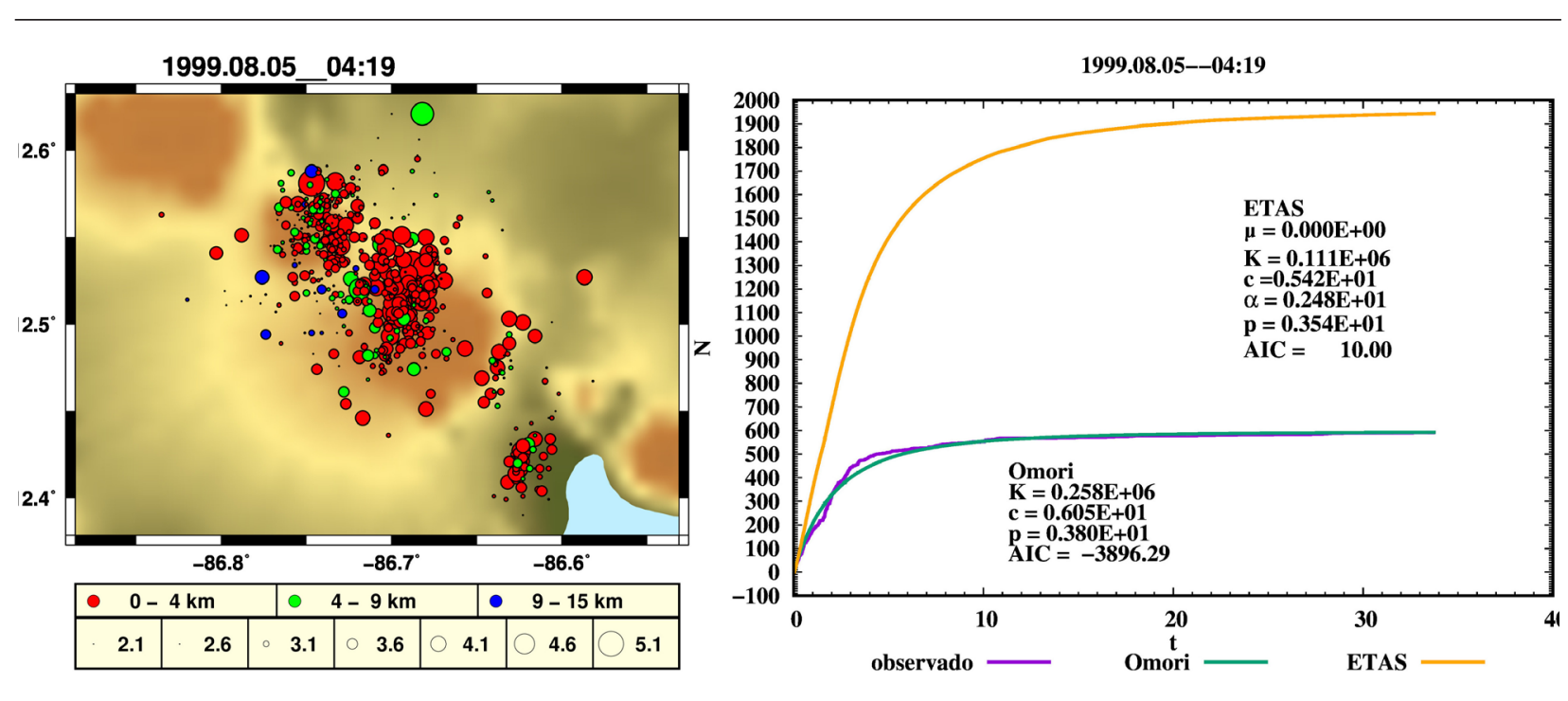

Figura 4. Agrupamiento de terremotos en los alrededores del volcán Cerro negro (1999). Fuente: elaboración propia en base a gnuplot" (Williams y Kelley, 2017) y el GMT (Wessel y Smith, 1998).

(2019) que analiza los seis primeros, mientras que otros estudian solo algunos, como es el caso de (Alvarez et al., 2018; French et al., 2010; La Femina et al., 2004). En este trabajo solo se analizarán algunas características adicionales a lo ya publicado. Se presentan de forma ilustrativa los mapas de epicentros y los gráficos del ajuste de ambos modelos estadísticos a las series temporales.

\section{a) Mes de agosto de 1999, volcán Cerro Negro}

Este proceso coincidió con una erupción del volcán Cerro Negro, y la relación entre ambos fenómenos fue estudiada por La Femina et al. (2004) y Segura (2019). En este último se hace un estudio muy detallado espacio temporal de la relación entre las actividades sísmica y eruptivas incluyendo los eventos de 1995. El proceso tuvo una $\mathrm{M}_{\mathrm{w}}$ equivalente de 5,78, comenzó el 5/8/1999 y tuvo una duración de 35 días. La caída de esfuerzos efectiva fue de 7,05 bar. Una característica interesante es que parecen existir tres fuentes independientes (NW, centro, SE) (figura 4). No obstante, la cantidad de eventos en cada una no permite estudiarlas por separado y el análisis estadístico se realizó para todos los terremotos. El ajuste por el modelo ETAS es muy malo y se desecha. 


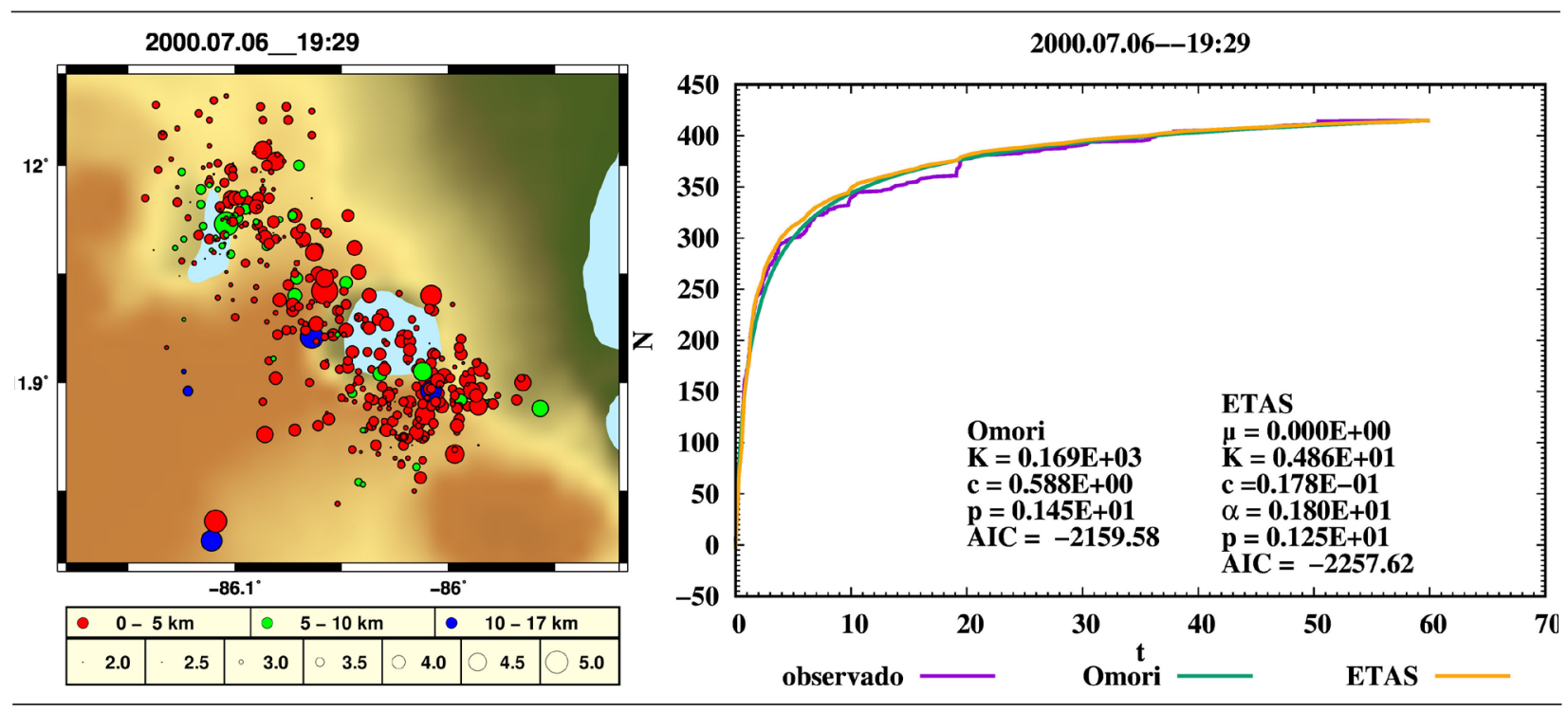

Figura 5. Agrupamiento de terremotos en la zona de la Laguna de Apoyo - Masaya (2000). Fuente: elaboración propia en base a gnuplot" (Williams y Kelley, 2017) y el GMT (Wessel y Smith, 1998).
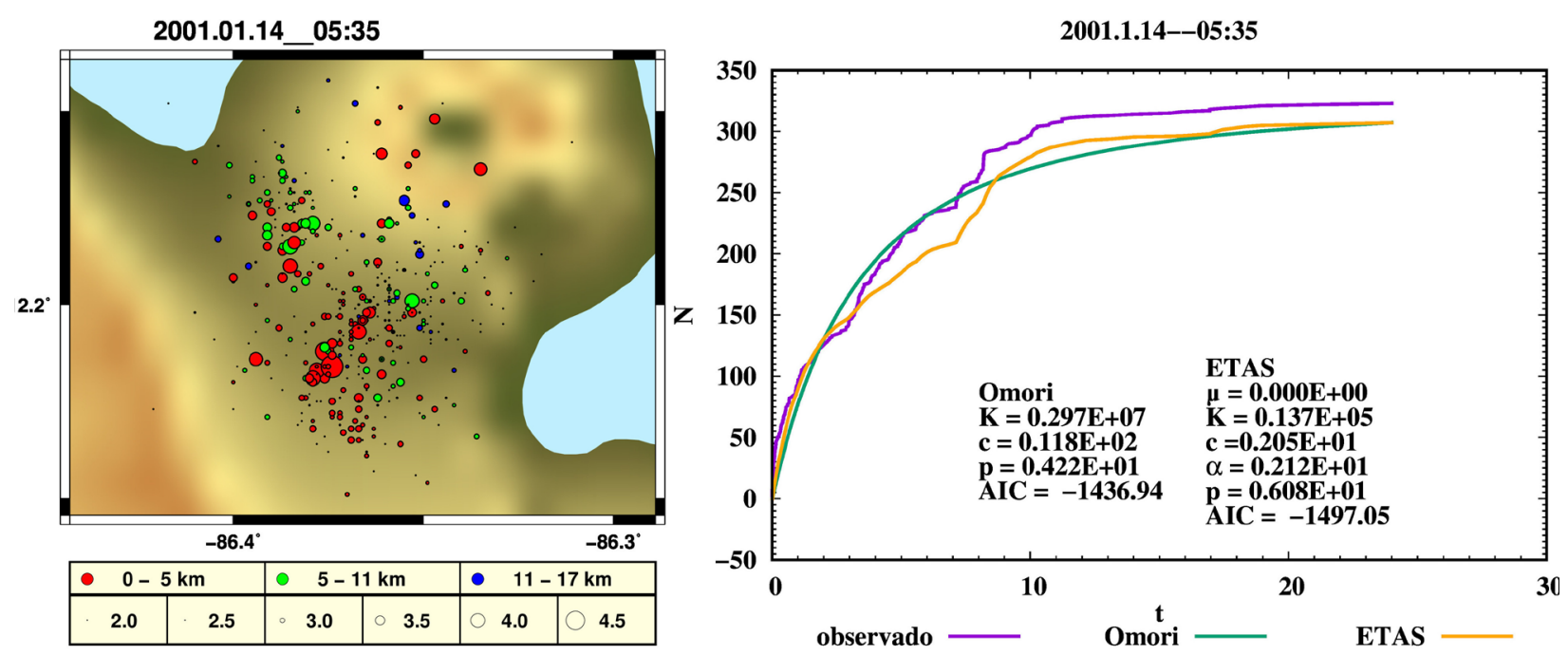

Figura 6. Agrupamiento de terremotos en la península de Chiltepe, lago Xolotlán (2001). Fuente: elaboración propia en base a gnuplot" (Williams y Kelley, 2017) y el GMT (Wessel y Smith, 1998).

b) Meses junio-agosto de 2000, Laguna de Apoyo, Departamento de Masaya

El proceso tuvo una $\mathrm{M}_{\mathrm{w}}$ equivalente de 5,79, comenzó el 6/7/2000 y tuvo una duración de 60 días. La caída de esfuerzos efectiva fue de 3,94 bar. En la figura 5 se nota, aunque con menor definición que en el caso anterior, la posible existencia de tres fuentes, que tampoco fue posible tratar por separado. Segura (2019) hace un análisis sobre la ocurrencia de los terremotos débiles y su relación con el fallamiento local. Son muy próximos los ajustes por el modelo de Omori modificado y el ETAS, pero el criterio de información de Akaike (1974), recomendado para este tipo de análisis (Ogata \& Katsura, 1985) simbolizado por AIC (Akaike Information Criterium) determina un mejor ajuste por el modelo ETAS.

c) Mes de enero 2001, península de Chiltepe, lago Xolotlán

El proceso tuvo una $\mathrm{M}_{\mathrm{W}}$ equivalente de 5,09, comenzó el 14/1/2001 y tuvo una duración de 27 días. La caída de esfuerzos efectiva fue de 7,33 bar. Segura (2019) presenta además algunas determinaciones de mecanismo focal de tipo corrimiento por el rumbo. El agrupamiento parece ser debido a una sola fuente y el mejor ajuste nuevamente es el que corresponde al modelo ETAS (el menor AIC) (figura 6). 


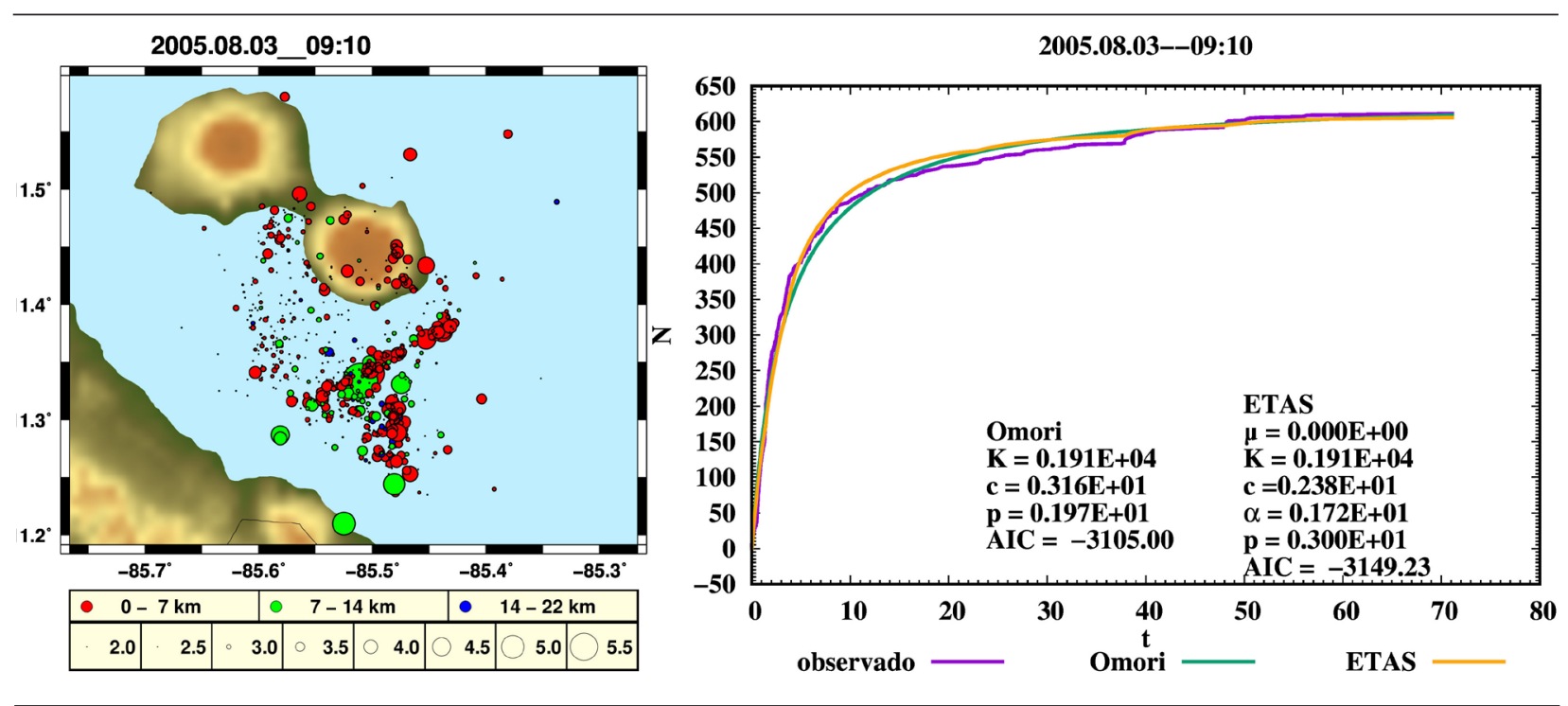

Figura 7. Agrupamiento de terremotos en el SE de la isla de Ometepe, lago Cocibolca (2005). Fuente: elaboración propia en base a gnuplot" (Williams y Kelley, 2017) y el GMT (Wessel y Smith, 1998).

La interpretación de este agrupamiento es dual: o bien es una serie donde el terremoto principal ocurre tres días después del inicio, o es un proceso PTR, con la ocurrencia de un premonitor fuerte, con una inmediata disminución de la cantidad de eventos y la ocurrencia a los tres días del terremoto principal y sus réplicas. Igualmente, según el AIC, el mejor ajuste es con el modelo ETAS.

d) Meses agosto-septiembre 2005, isla de Ometepe, lago Cocibolca

Este agrupamiento de terremotos fue objeto de un estudio especial que incluyó la instalación de una red de sismómetros portátiles para la localización precisa de las réplicas (French et al., 2010). El proceso tuvo una $M_{w}$ equivalente de 6,32, comenzó el 3/8/2005 y tuvo una duración de 71 días. La caída de esfuerzos efectiva fue de 2,85 bar. El proceso comenzó con algunos premonitores, incluso uno bastante fuerte $\left(\mathrm{M}_{\mathrm{w}}=5,3\right)$, seguida casi inmediatamente por el terremoto principal. French et al. (2010) identifican dos fuentes diferentes, una de dirección NE-SW y otra de dirección N-S, a la que se puede sumar una tercera con sismicidad más difusa en la mitad SE de la isla de Ometepe (figura 7). En este caso el mejor ajuste resulta también con el modelo ETAS.

\section{e) Meses abril-julio 2014 mitad occidental del lago Xolotlán}

Este agrupamiento de terremotos ha sido muy estudiado, y se destacan los trabajos de Moreno et al. (2014), INETER (2014) y Alvarez et al. (2018). El proceso tuvo una $\mathrm{M}_{\mathrm{w}}$ equivalente de 6,21, comenzó el 10/4/2014 y tuvo una duración de 137 días. La caída de esfuerzos efectiva fue de 3,42 bar. En este caso se ve claramente una fuente aislada en la península de
Chiltepe, y se perfila una división del resto del agrupamiento en dos fuentes, una sobre el lago (volcán Momotombito) y otra al NW (volcán Momotombo) (figura 8). El análisis por separado de las mismas muestra que solo la zona central tiene un comportamiento PTR, mientras que las otras dos no se ajustan a ese modelo, por lo que se decidió considerar la serie como un todo para el análisis estadístico. Aquí, igualmente, el mejor ajuste se obtiene con el modelo ETAS.

\section{f) Meses septiembre-noviembre 2015, El Sauce, Departamento de León}

Este agrupamiento de terremotos ha sido bastante estudiado también (Alvarez et al., 2018; González et al., 2015). Es una serie de terremotos de magnitud relativamente baja, muy importante por ocurrir en el borde este de la depresión de Nicaragua. El proceso tuvo una $M_{w}$ equivalente de 5,38, pero el evento máximo solo alcanzó $\mathrm{M}_{\mathrm{w}}=4,7$. Comenzó el 10 de abril de 2014 y tuvo una duración de 137 días. La caída de esfuerzos efectiva fue de 2,51 bar. En este caso, aunque el mejor ajuste según el AIC corresponde al modelo ETAS, a simple vista parece mejor el de Omori modificado (figura 9).

\section{g) Meses junio-agosto de 2016, Puerto Morazán, Departamento de Chinandega}

Este agrupamiento de terremotos ha sido estudiado previamente por Segura \& Alvarez (2016) y Alvarez et al. (2018). El proceso tuvo una $M_{w}$ equivalente de 6,18, comenzó el 10/6/2016 y tuvo una duración de 86 días. La caída de esfuerzos efectiva fue de 2,05 bar. Se identifica una sola zona epicentral y el mejor ajuste se obtiene con el modelo ETAS (figura 10). 

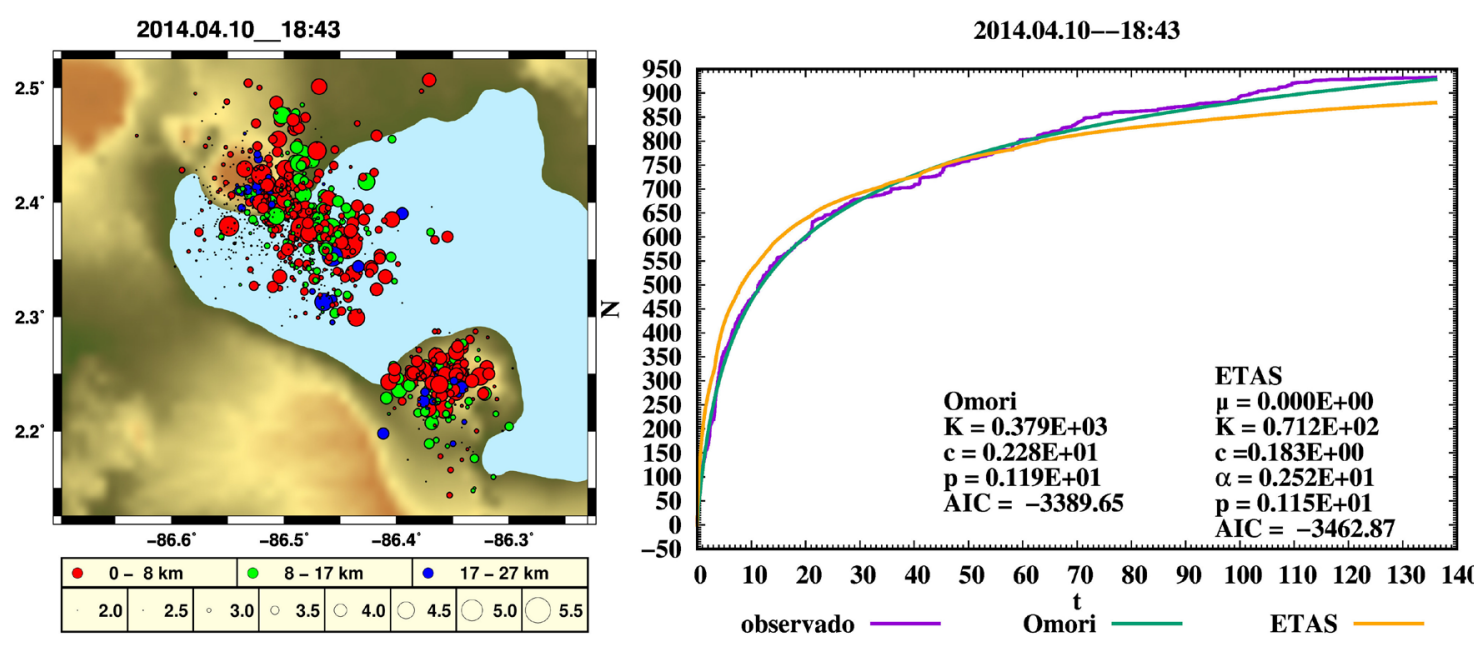

Figura 8. Agrupamiento de terremotos en la mitad occidental del lago Xolotlán (2014). Fuente: elaboración propia en base a gnuplot" (Williams y Kelley, 2017) y el GMT (Wessel y Smith, 1998).
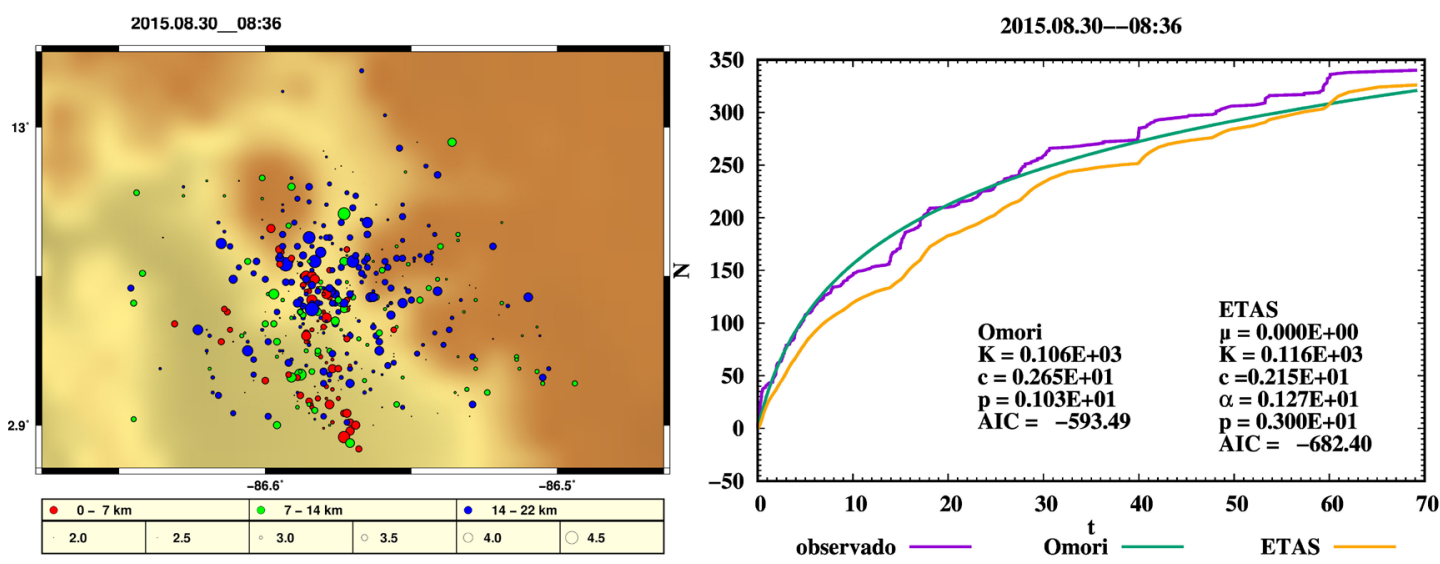

Figura 9. Agrupamiento de terremotos en la zona de El Sauce, Departamento de León (2015). Fuente: elaboración propia en base a gnuplot" (Williams y Kelley, 2017) y el GMT (Wessel y Smith, 1998).
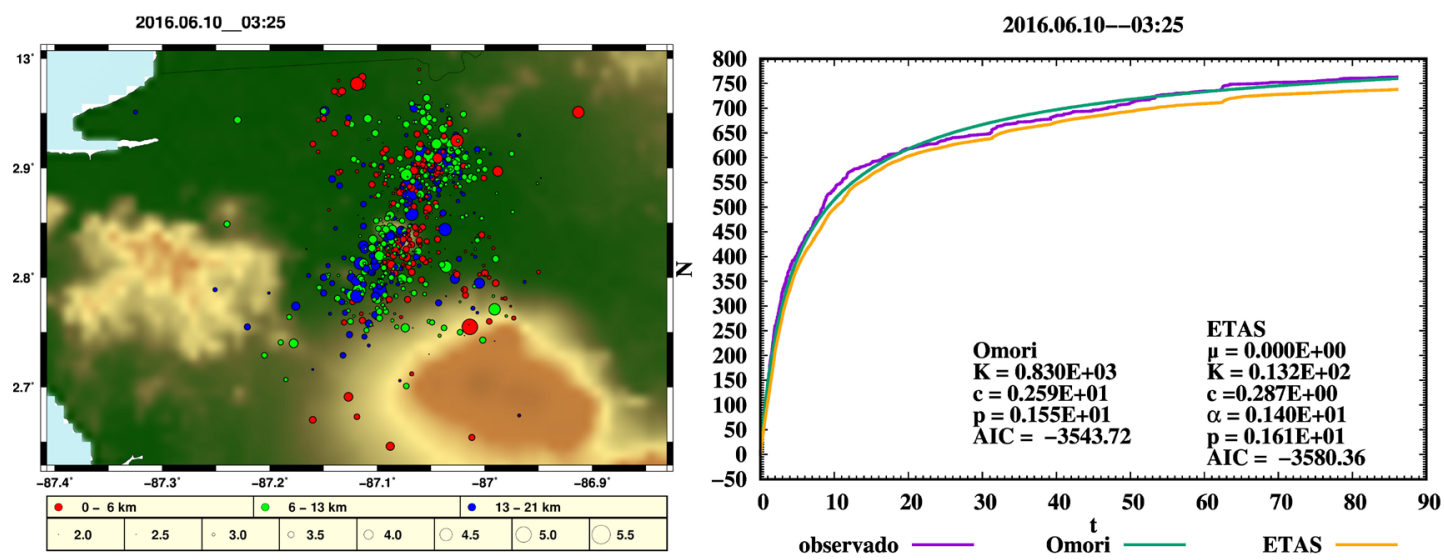

Figura 10. Agrupamiento de terremotos en la zona de Puerto Morazán, Departamento de Chinandega (2016). Fuente: elaboración propia en base a gnuplot" (Williams y Kelley, 2017) y el GMT (Wessel y Smith, 1998). 

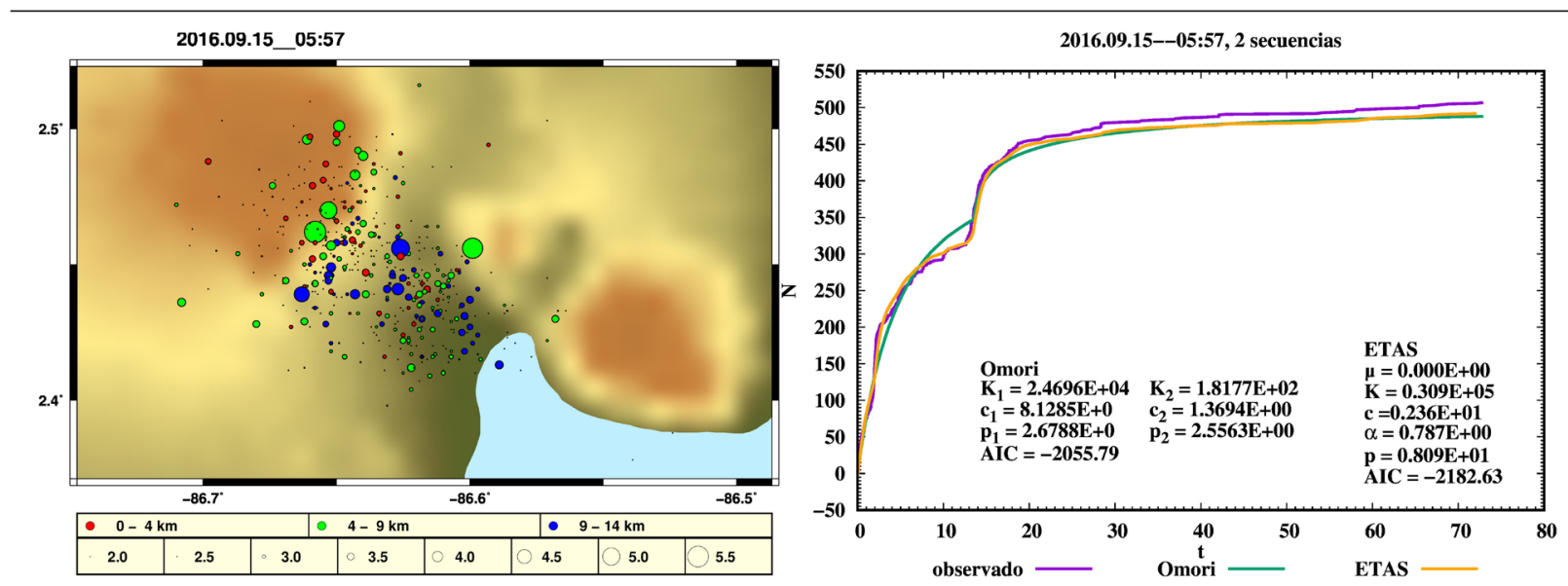

Figura 11. Agrupamiento de terremotos en la zona del volcán El Hoyo (2016). Fuente: elaboración propia en base a gnuplot" (Williams y Kelley, 2017) y el GMT (Wessel y Smith, 1998).

\section{h) Meses septiembre-octubre de 2016, volcán El Hoyo}

Este agrupamiento de terremotos tiene una característica peculiar, y es que pertenece al tipo que responde a un modelo de Omori modificado con dos secuencias (Alvarez \& Segura, 2018; Alvarez et al., 2018). Este tipo de comportamiento también es ajustable por el modelo ETAS (figura 11). El proceso tuvo una $\mathrm{M}_{\mathrm{w}}$ equivalente de 5,78, comenzó el 15/9/2016 y tuvo una duración de 53 días. La caída de esfuerzos efectiva fue de 7,20 bar. El evento principal con $\mathrm{M}_{\mathrm{w}}=5,6$ inició el proceso, y el evento que inició la segunda secuencia ocurrió el 28 de septiembre, con $\mathrm{M}_{\mathrm{W}}=5,4$. La primera secuencia está concentrada mayormente en la mitad NW del área epicentral y la segunda en la mitad SE.

\section{i) Análisis comparativo}

Debe señalarse que, con otros algoritmos de selección de eventos agrupados, o con el mismo algoritmo, pero usando otros parámetros, la composición de los agrupamientos podría variar. Otro elemento de variación está relacionado con el cálculo de la caída de esfuerzos efectiva. El algoritmo usado considera todos los eventos del agrupamiento para calcular su área (y consiguientemente el valor de $R$ en la fórmula 14). Si se eliminan algunos eventos alejados del núcleo principal del agrupamiento, generalmente bastante más débiles, el valor de $\Delta \tau_{\text {efec }}$ debería aumentar. Esto debe tenerse en cuenta para efectuar estudios detallados de agrupamientos específicos.

Es importante señalar que, con excepción del proceso en 1999 en las inmediaciones del volcán Cerro Negro, el modelo ETAS es el que mejor se ajusta a los datos experimentales usando el AIC, aunque su diferencia con el modelo de Omori (para casos de una o dos secuencias) no es muy grande. Esto hace suponer que en procesos futuros tipo PTR y S se debe cumplir también y se pueden usar las características predictivas de ambos modelos durante su análisis en tiempo real. Otro aspecto importante para destacar es la influencia de la calidad de las localizaciones epicentrales en la definición de posibles fuentes principales de terremotos. Se ve que en los últimos cinco casos donde se realizó un trabajo de relocalización de hipocentros (los más detallados para los agrupamientos de 2005 y 2014) esta definición es mucho más evidente que en los primeros tres.

\section{2) Caracterización espacio-temporal-energética de la sismicidad}

La región de estudio es la señalada en la figura 1 como límite del catálogo. Se diferencian tres macrozonas por intervalos de profundidad: $[0,40) \mathrm{km},[40,110) \mathrm{km}$ y $[110,250) \mathrm{km}$. Las dos últimas se analizan como un todo, mientras que la primera se divide en tres partes: océano Pacífico hasta el inicio de la cadena volcánica de Centroamérica, la propia cadena volcánica y desde esta última hasta los límites en el mar Caribe. La cadena volcánica se subdivide en 3 partes, un pequeño sector en El Salvador, la depresión de Nicaragua y una zona un poco más amplia en Costa Rica (figura 12). Esta última división está motivada por las características particulares de la sismicidad en la depresión de Nicaragua que han sido estudiadas por Alvarez et al. (2018). No obstante, debe señalarse que esta zonación es bastante general y responde a las características del comportamiento de la sismicidad (figura 3), pero dista mucho de ser la que se 
Tabla 5

Parámetros que caracterizan la sismicidad de las zonas fuente.

\begin{tabular}{|c|c|c|c|c|c|}
\hline Zona & a & bb & $M_{\max }$ (obs) & $\mathbf{M}_{\max }{ }^{(K \mathrm{Kj} k 0)}$ & otras $M_{\max }$ \\
\hline Pacífico & 8,16 & 1,310 & 7,6 & $7,64 \pm 0,20$ & $7,0-8,1\left(^{3}\right)$ \\
\hline cadena & 5,295 & 0,958 & $6,4\left(^{1}\right)$ & $6,46 \pm 0,21$ & 5,5 (borde interior), 6,5 (centro), 5 (borde exterior) $\left(^{2}\right), 6,7-7,8\left({ }^{3}\right)$ \\
\hline eSalv & 5,121 & 1,060 & 7,0 & $7,30 \pm 0,36$ & $6,4-7,1\left(^{3}\right)$ \\
\hline cRica & 5,379 & 0,972 & 7,6 & $7,80 \pm 0,28$ & $6,4-7,8\left({ }^{3}\right)$ \\
\hline este & 6,169 & 1,128 & 6,3 & $6,34 \pm 0,20$ & $5,9-6,5\left(\left(^{3}\right)\right.$ \\
\hline$h=[40,110) \mathrm{km}$ & 7,355 & 1,208 & 7,7 & $7,75 \pm 0,21$ & $7,4-7,9\left({ }^{3}\right)$ \\
\hline$h=[110,250) \mathrm{km}$ & 7,182 & 1,261 & 7,2 & $7,31 \pm 0,23$ & \\
\hline
\end{tabular}

(1) Existe un terremoto con $M W=6,9$ que no se tomó en consideración; ocurrió el 5/11/1926 y el GEM lo sitúa dentro de esta zona. Sin embargo, este terremoto se sintió muy fuerte en toda Nicaragua y otros lugares de Centroamérica, por lo que se asumió que sus coordenadas eran erróneas y debía asociarse a la zona Pacífico; (2) estimaciones de Alvarez et al. (2018); ( ${ }^{3}$ ) estimaciones de Alvarado et al. (2017).

Fuente: elaboración propia.

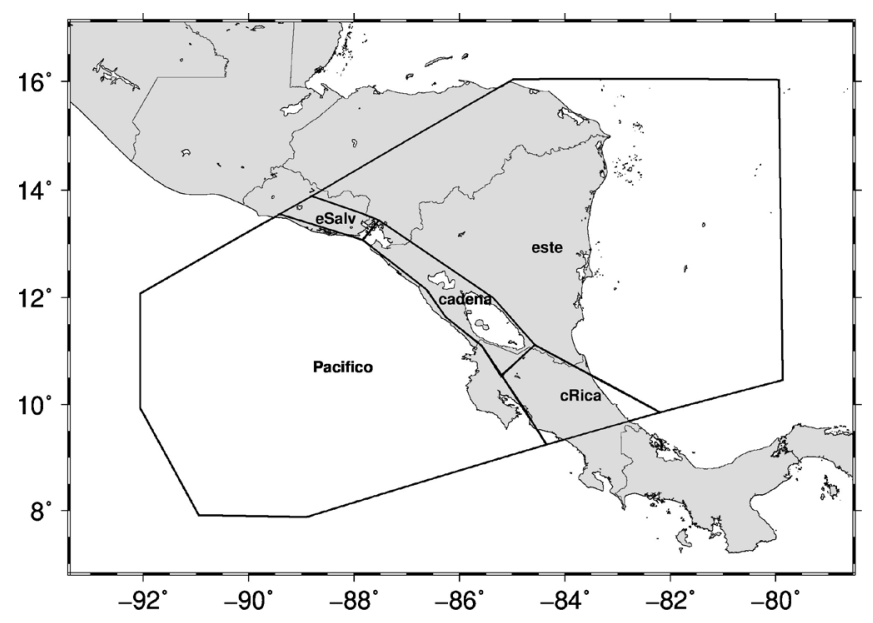

Figura 12. División de la región en zonas de ocurrencia de terremotos superficiales $[0,40) \mathrm{km}$. Fuente: elaboración propia en base a gnuplot" (Williams y Kelley, 2017) y el GMT (Wessel y Smith, 1998).

usaría en un trabajo de amenaza sísmica (Alvarado et al., 2017; Alvarez, 2021).

La zona "Pacifico", al igual que las zonas de terremotos de profundidades mayores (en su mayoría) corresponden al proceso de subducción; las zonas "eSalv" y "cadena" corresponden a los procesos que ocurren a lo largo de la cadena volcánica (El Salvador - Nicaragua), la zona "cRica" comprende una parte de Costa Rica de procesos tectónicos complejos, mientras que en la zona "este" se engloban diversas fuentes de terremotos de interior de placa.

En la tabla 5 se presentan los valores de los parámetros $(a, b)$ de los gráficos magnitud frecuencia, así como diversas estimaciones de $M_{\max }$ para cada una de las zonas de la figura 12. Para obtener los valores de $(\mathrm{a}, \mathrm{b})$ se usaron los intervalos de representatividad presentados en Alvarez (2021) y los gráficos distributivos experimentales fueron ajustados por el método de la máxima verosimilitud. Los valores de la magnitud máxima observada se determinaron a partir del catálogo de terremotos. Este es un proceso complicado, pues entra en juego la confiabilidad de los estimados de coordenadas y magnitud. En los primeros años de registro instrumental, con muy pocas estaciones trabajando, el error en las coordenadas es muy grande. Se tomó la decisión de considerar el catálogo a partir de 1929, obviando los años anteriores. Si bien es cierto que el GEM ha realizado un trabajo muy riguroso en la reestimación de las magnitudes de los terremotos fuertes, por la falta de datos no se ha logrado una precisión buena en las coordenadas de los terremotos en las primeras tres décadas del siglo xx. Un ejemplo de esto es el terremoto 


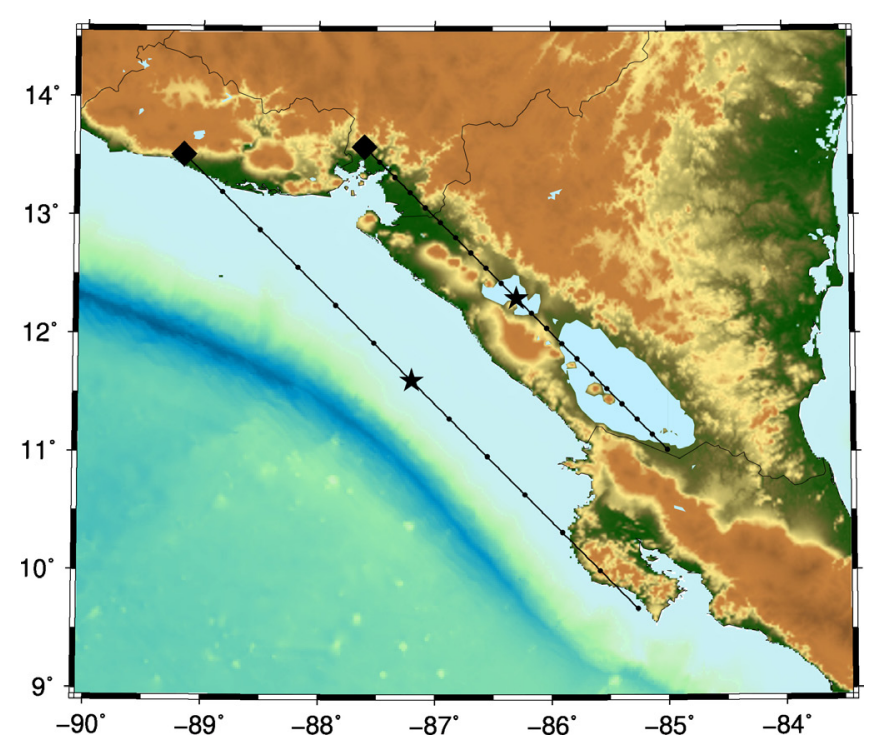

Figura 13. Perfiles para el análisis espacio temporal. Fuente: elaboración propia en base a gnuplot" (Williams y Kelley, 2017) y el GMT (Wessel y Smith, 1998).

de Managua de 1931, con epicentro estudiado con trabajos de campo en el área de Managua, que el GEM sitúa a unos $200 \mathrm{~km}$ de distancia en la zona de subducción frente a Chinandega. De forma individual se han analizado algunos casos, que se comentan en la tabla 5.

Para el cálculo de $\mathrm{M}_{\max }$, se usó el programa “AUE” (Kijko, 2020). Entre las opciones de métodos disponibles para determinar la $\mathrm{M}_{\max }$ se usó la fórmula bayesiana de KijkoSelevoll (Kijko \& Selevoll, 1989; Kijko \& Singh, 2011). En este caso se utilizó igualmente el catálogo desde 1929. Alvarado et al. (2017) subdividen la región de estudio en múltiples zonas pequeñas y para cada una usan tres métodos de estimación; para la Tabla 5 se tomaron los valores máximos y mínimos de todas las estimaciones para las subzonas que comprenden una de las zonas usadas en este trabajo.

Los gráficos magnitud frecuencia no permiten pueden particularizar en las características espacio-temporales de la sismicidad dentro de una zona, pues, se caracteriza cada zona por solo tres parámetros. Hay dos aspectos muy útiles para conseguir el objetivo de caracterizar completamente la ocurrencia de terremotos, y son el comportamiento temporal de la liberación de energía, y la representación en tiempo y espacio de la ocurrencia de terremotos. Este análisis se realizó para las dos zonas que tienen más influencia sobre las ciudades del Pacífico de Nicaragua ("Pacifico" y "cadena"). Para ambos casos se prepararon gráficos de liberación de energía acumulativa y distributiva y un gráfico espacio-temporal. Este último se realizó a lo largo de dos perfiles (figura 13).

Se consideró el catálogo de forma orientadora desde el año 1905, aunque en realidad los resultados son confiables solo a partir de 1929. En el caso de la zona "cadena" se seleccionaron las magnitudes superiores a $M_{W}=5$, y para el caso "Pacifico" a partir de $M_{W}=6$. La energía se evalúa por la fórmula (20) usando $M_{W}$ en lugar de $M_{S}$.

Una combinación de gráficos de liberación de energía con gráfico espacio-temporal se muestra en las figuras 14 (Pacífico) y 15 (depresión de Nicaragua - zona cadena en figura 12).

En la figura14 se observa que hay episodios de incremento de la energía liberada con intervalos entre 20 y 40 años. Desde el punto de vista espacial se observa una zona entre los 100 y $250 \mathrm{~km}$ al sureste del centro del perfil donde no han ocurrido eventos fuertes desde antes de 1920, por lo que es la zona con más probabilidad de que ocurra un evento de ese tipo en el futuro mediato. Debe señalarse que tal ausencia de terremotos fuertes en esa zona se extiende hasta la profundidad de $250 \mathrm{~km}$. En el extremo noroeste se observa también una zona "vacía". Independientemente de que el análisis de esta zona requeriría considerar una región un poco más amplia hacia El Salvador, no incluida en el catálogo, debe señalarse que por varios años el Instituto 


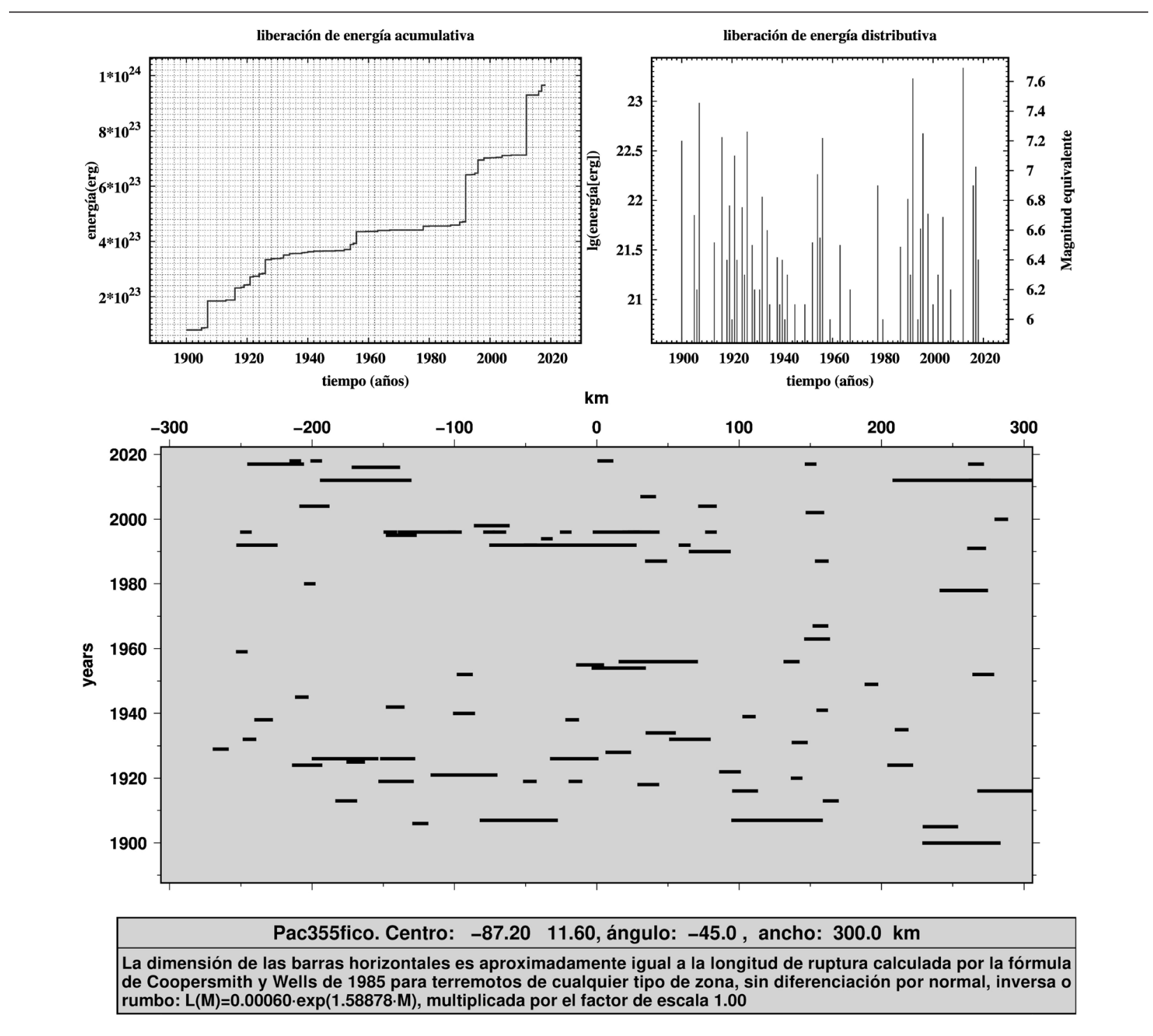

Figura 14. Caracterización espacio-temporal de la sismicidad en la zona "Pacífico". Fuente: elaboración propia en base a gnuplot" (Williams y Kelley, 2017) y el GMT (Wessel y Smith, 1998).

Internacional de Teoría del Pronóstico de Terremotos y Geofísica Matemática ${ }^{1}$ estuvo señalando esa zona como de probabilidad incrementada de ocurrencia de un evento con $\mathrm{M}_{\mathrm{w}}>7,5$, aunque luego retiró la alerta.

En la figura 15 se observa también cierta ciclicidad en los máximos de liberación de energía, no tan acusada como en el caso anterior, con períodos de entre 20 y 30 años. Igualmente, hay dos zonas que no han experimentado eventos fuertes desde hace más de 60 años y están ubicadas entre
50 y $90 \mathrm{~km}$ hacia el noroeste del centro del perfil, y entre los 90 y $120 \mathrm{~km}$ hacia el suroeste de este. En ellas es más probable que ocurran tales eventos en el futuro mediato. La depresión de Nicaragua fue objeto de un análisis especial por Alvarez et al. (2018). Dichos autores plantearon un modelo sismotectónico, estimaron magnitudes máximas posibles y señalaron dos zonas de posible ocurrencia mediata de eventos de hasta $\mathrm{M}_{\mathrm{w}}=6,5$ (figura 16), que se corresponden con las identificadas en la figura 15 como más propensas a la ocurrencia de eventos fuertes en un futuro mediato. 


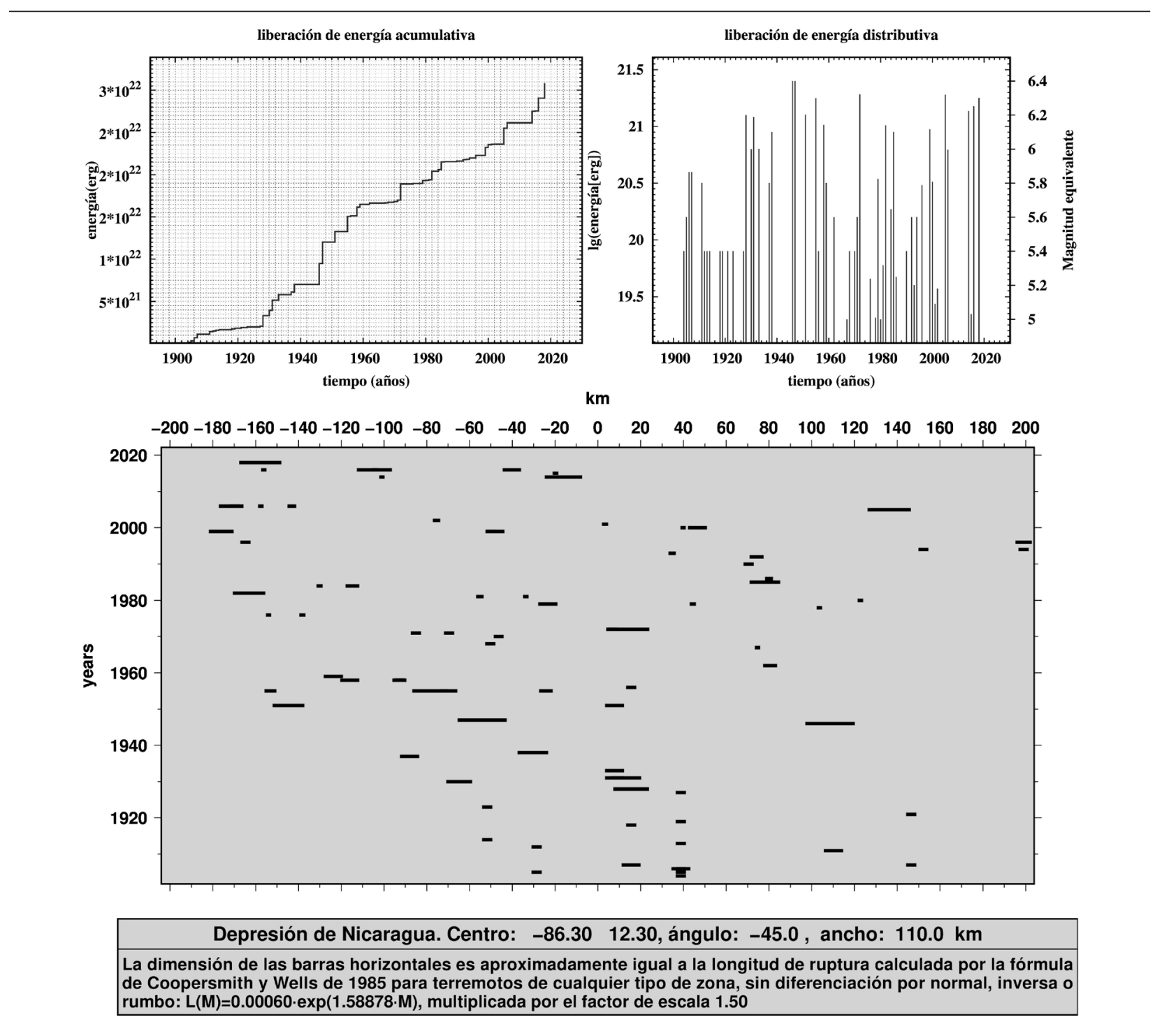

Figura 15. Caracterización espacio-temporal de la sismicidad en la depresión de Nicaragua. Fuente: elaboración propia en base a gnuplot" (Williams y Kelley, 2017) y el GMT (Wessel y Smith, 1998).

La figura 16 resume los planteamientos de Alvarez et al. (2018) y merece ser explicada. Los eventos ocurren en una serie de fallas escalonadas con dirección NE-SW, que giran hacia N-S en sus extremos y se caracterizan por los mecanismos focales que se indican en el recuadro superior derecho. La zona de la depresión de Nicaragua (línea discontinua) es más ancha y algo menos extensa que la zona "cadena" usada en el análisis de este trabajo. Los círculos rojos corresponden a epicentros de terremotos significativos desde 1972. Las magnitudes máximas están representadas esquemáticamente por la división en zonas indicada por las líneas de puntos: $\mathrm{M}_{\max }=6,5$ en la parte central, $\mathrm{M}_{\max }=5,5$ en el borde con la zona del este de
Nicaragua y $\mathrm{M}_{\max }=5,0$ en el borde con el Pacífico. Los límites de esta división se trazaron en el presente trabajo de forma convencional, pues los autores no lo hicieron. De hecho, consideramos que no hay datos suficientes para precisarla más. Finalmente, las líneas gruesas en zonas sin epicentros son los lugares que los autores señalan como de mayor probabilidad de ocurrencia de eventos en el futuro mediato.

\section{Discusión y conclusiones}

- Se confeccionó en catálogo de terremotos caracterizado por "la mejor localización disponible" y el máximo 


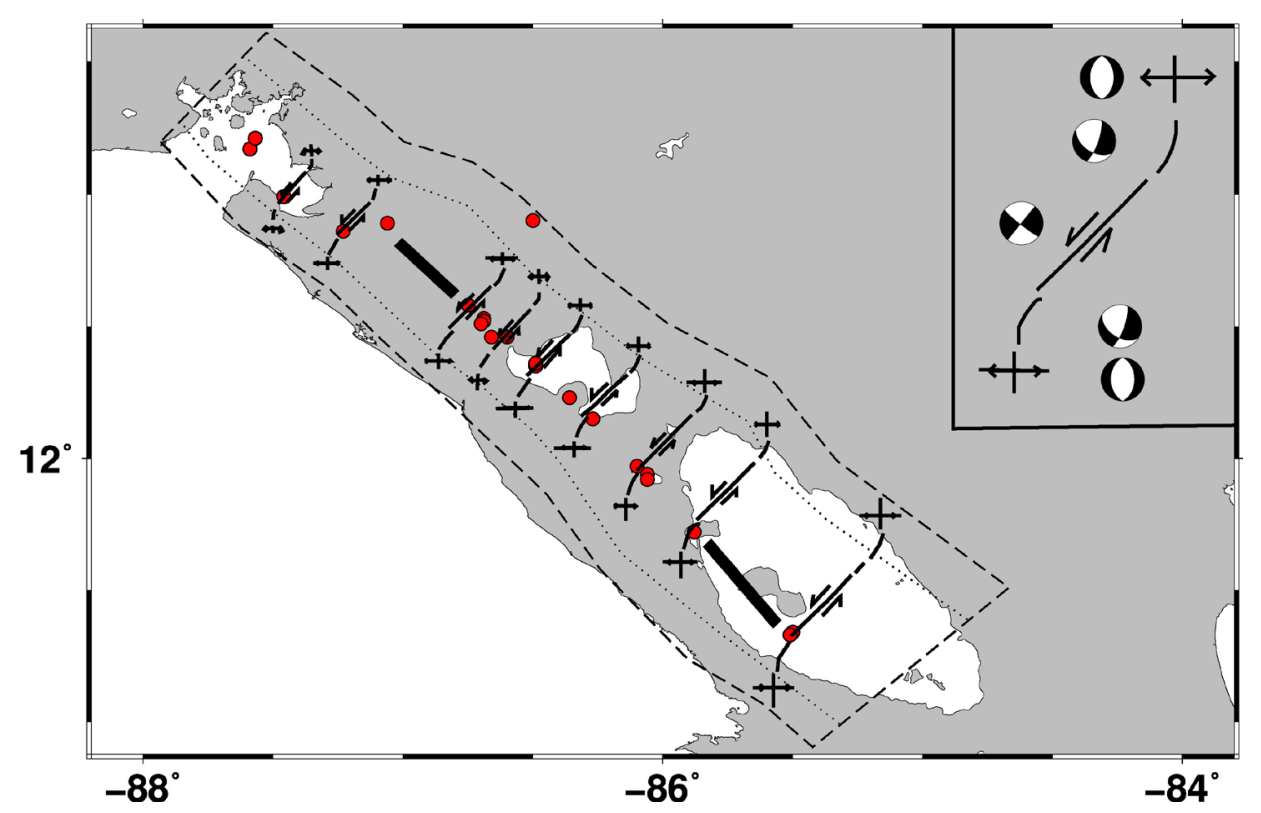

Figura 16. Sismotectónica y pronóstico a mediano plazo. Modificado de Alvarez et al. (1998). Fuente: elaboración propia en base a gnuplot" (Williams y Kelley, 2017) y el GMT (Wessel y Smith, 1998).

posible de magnitudes. El catálogo confeccionado se considera como la mejor opción posible para el nivel de conocimiento actual. Contiene 76.935 terremotos, de los que 74.500 tienen al menos una determinación de magnitud. Sin embargo, no se puede considerar como finalizado por dos motivos. En primer lugar, continúan realizándose relocalizaciones de eventos, ya sean débiles o fuertes que deben actualizarse. En segundo lugar, la inmensa mayoría de los eventos tienen sus coordenadas determinadas por la red de Nicaragua, lo que depende grandemente del modelo de corteza utilizado. Si en algún momento se cambia el modelo de corteza a uno más adecuado deben recalcularse todas las coordenadas de los eventos. Finalmente está el análisis casuístico de los eventos más fuertes de las primeras décadas del siglo XIX. La escasez de estaciones usadas en las localizaciones hace que tengan grandes errores y puede ser necesario corregir sus coordenadas usando nuevos datos de efectos sentidos u otras consideraciones.

- Se preparó una versión de este unificada a $\mathrm{M}_{\mathrm{w}}$, para lo cual se establecieron relaciones entre los distintos tipos de magnitud presentes y la $\mathrm{M}_{\mathrm{w}}$. Algunas de ellas podrían variar en el futuro si con la adquisición de nuevos datos se obtuvieran relaciones de conversión $\mathrm{M}_{\mathrm{i}} \rightarrow \mathrm{M}_{\mathrm{W}}$ más precisas.

- Se realizó un análisis de eventos agrupados que permitió individualizar los grupos de terremotos para su análisis estadístico y preparar un catálogo sin eventos relacionados para el análisis de la sismicidad.

- Se analizaron ocho grupos de terremotos que incluyen procesos terremoto-principal-réplicas y series y se determinó que, con excepción de uno, el mejor ajuste se obtiene con el modelo ETAS, aunque el ajuste con la ley de Omori modificada (con una o dos secuencias) es bastante próximo, y el único posible para la excepción (volcán Cerro Negro, 1999). El ajuste de modelos estadísticos suele ser una herramienta muy útil para pronosticar el comportamiento de las secuencias de terremotos en el futuro. Este tipo de eventos es muy común en la depresión de Nicaragua (Segura, 2019) y tienen una gran influencia en la vida del país. Se puede hacer mucho más en este sentido, pero va más allá de los objetivos del presente trabajo.

- Usando una versión del catálogo sin eventos relacionados se estudiaron los parámetros del régimen sísmico de siete zonas en que se dividió convencionalmente la región de estudio, estimándose los parámetros $(a, b)$ de los gráficos magnitud frecuencia, la $\mathrm{M}_{\max }$ observada y la $\mathrm{M}_{\max }$ calculada por el método de Kijko, lo que constituye una buena caracterización de la sismicidad de la región de estudio. Estas zonas tienen un grado de generalidad grande, y no es objeto del presente trabajo la discusión de sus límites específicos, lo que corresponde a las tareas de una estimación de la peligrosidad sísmica. 
- El análisis espacio-temporal de la ocurrencia de terremotos permitió definir comportamientos cíclicos en la ocurrencia de eventos fuertes, lo que conllevó al reconocimiento de los lugares donde pueden ocurrir tales eventos en un futuro mediato. Para la zona de la depresión de Nicaragua y la subducción en el Pacífico se analizó la ciclicidad de la ocurrencia de terremotos fuertes (20-30 años en la depresión, 20-40 años en el Pacífico).

- Se identificaron las zonas con mayor probabilidad de ocurrencia de terremotos fuertes en un futuro mediato (dos zonas en la depresión (entre las islas Zapatera y Ometepe, y entre el volcán Rota y la ciudad de Tonalá) y una en el Pacífico (desde Rivas hacia Costa Rica). Las magnitudes máximas posibles son del orden de 6,5 para las zonas en la depresión y 7,8 en la zona del Pacífico

\section{Materiales adicionales}

El catálogo de terremotos y algunas tablas adicionales están accesibles en: http://iohanis.com/ leoalvar/datos/ CatalogoNicaragua.zip

\section{Referencias}

Akaike, H. (1974). A new look at the statistical model identification. IEEE Trans. Automat. Control, 19(6), 716-723. https://doi.org/10.1109/TAC.1974.1100705

Algermissen, S.T., Dewey, J.W., Langer, C.J. \& Dillinger, W.H. (1974). The Managua, Nicaragua, earthquake of December 23,1972: Location, focal mechanism, and intensity distribution. Bull. Seism. Soc. of America, 64(4), 993-1004. https://doi. org/10.1785/BSSA0640040993

Alvarado, G.E.., Benito, B., Stallerb, A., Climent, A., Camacho, E., Rojas, W., Marroquín, G., Molina, E., Talavera, E., Martínez-Cuevas, S. \& Lindholm, C. (2017). The new Central American seismic hazard zonation: Mutual consensus based on up to day seismotectonic framework. Tectonophysics, 721, 462-476. http://doi.org/10.1016/j.tecto.2017.10.013

Alvarez, L. (2021). Nueva estimación de la amenaza sísmica para Nicaragua. Revista Tierra, 1(2). https:// revistatierra.unan.edu.ni.
Alvarez, L., Lindholm, C. \& Villalón, M. (2017). Seismic Hazard for Cuba: A New Approach. Bull. Seism. Soc. of America, 107(1), 229-239. https://doi. org/10.1785/0120160074

Alvarez, L. \& Segura, F. (2016). Sismicidad de la zona de ocurrencia de los terremotos. Bol. Sismos y Volcanes de Nicaragua, 17-24. https://webserver2. ineter.gob.ni/sis/bolsis/bolsis.html

Alvarez, L., Rodríguez, A.M., González, O., Moreno, B. \& Cabrera, A. (2018). Seismotectonics of the Nicaraguan Depression from Recent Seismicity. Journal of Geology \& Geophysics, 7(5). https://doi. org/10.4172/2381-8719.1000446

Bakun, W.H. (1984). Seismic moments, local magnitudes, and coda-duration magnitudes for earthquakes in central California. Bulletin of the Seismological Society of America, 74(2), 439-458. https://doi.org/10.1785/BSSA0740020439.

Brax, M., Albini, P., Beauval, C., Jomaa, R. \& Sursock, A. (2019). An Earthquake Catalog for the Lebanese Region. Seismological Research Letters, 90(6), 22362249. https://doi.org/10.1785/0220180292

Beauval, C., Yepes, H., Palacios, P., Segovia, M., Alvarado, A., Font, Y., Aguilar, J., Troncoso, L. \& Vaca, S. (2013). An earthquake catalog for seismic hazard assessment in Ecuador. Bulletin of the Seismological Society of America, 103(2A), 773786. https://doi.org/10.1785/0120120270

Behr, Y., Clinton, J.F.., Cauzzi, C.., Hauksson, E., Jónsdóttir, K., Craiu G. Marius, C.G., Pinar, A., Salichon, J. \& Sokos, E. (2016). The Virtual Seismologist in SeisComP3: A New Implementation Strategy for Earthquake Early Warning Algorithms. Seismological Research Letters, 87(2A), 363-373. https://doi.org/10.1785/0220150235

Benito, M.B., Lindholm, C., Camacho, E., Climent, A., Marroquín, G., Molina, E., Rojas, W., Escobar, J.J., Talavera, E., G. E. Alvarado, G.E. \& Torres, Y. (2012). A New Evaluation of Seismic Hazard for the Central America Region. Bulletin of the Seismological Society of America, 102(2), 504-523. https://doi.org/10.1785/0120110015

Benito, B. \& Torres, Y. (edit.). (2009). Amenaza sísmica en América Central. Entimema. 
Bondár, I., Engdahl, E.R.., Villaseñor, A., Harris, J. \& Storchak, D.A. (2015). ISC-GEM: Global Instrumental Earthquake Catalogue (1900-2009): II. Location and seismicity patterns. Physics of The Earth and Planetary Interiors, 239(6), 2-13. https:// doi.org/10.1016/j.pepi.2014.06.002

Bondár, I. \& Storchak, D. (2011). Improved location procedures at the International Seismological Centre. Geophysical Journal International, 186(3), 1220-1244. https://doi.org/10.1111/j.1365246X.2011.05107.x

Bormann, P. (Edit.) (2002). IASPEI New Manual of Seismological Observatory Practice (NMSOP), Volume 1. GeoForschungsZentrum, Potsdam.

Cheng, J., Rong, Y., Magistrale, H., Guihua Chen, G. \& Xu, X. (2017). An Mw-Based Historical Earthquake Catalog for Mainland China. Bulletin of the Seismological Society of America, 107(5), 24902500. https://doi.org/10.1785/0120170102

Chuy, T. (1985). Datos macrosísmicos de la República de Nicaragua. Inv. Sismol. en Cuba, 5, 95-142.

Dewey, J.W. \&. Algermissen, S.T. (1974). Seismicity of the Middle America arc-trench system near Managua, Nicaragua. Bulletin of the Seismological Society of America, 64(4), 1033-1048. https://doi.org/10.1785/ BSSA0640041033

Di Giacomo, D., Bondár, I., Storchak, D.A., Engdahl, E.R., Bormann, P. \& J. Harris (2015). ISC-GEM: Global Instrumental Earthquake Catalogue (1900-2009): III. Re-computed MS and mb, proxy MW, final magnitude composition and completeness assessment. Physics of the Earth and Planetary Interiors, 239, 33-47. https:// doi.org/10.1016/j.pepi.2014.06.005

Di Giacomo, D., Engdahl, E.R. \& Storchak, D.A. (2018). The ISC-GEM Earthquake Catalogue (19042014): status after the Extension Project. Earth System Science Data, 10(4), 1877-1899. https://doi. org/10.5194/essd-10-1877-2018

Dziewonski, A.M., Chou, T.A. \& J. H. Woodhouse, J.H. (1981). Determination of earthquake source parameters from waveform data for studies of global and regional seismicity. Journal of Geophysical Research, 86(B4), 2825-2852. https://doi. org/10.1029/JB086iB04p02825.
Engdahl, E.R., Di Giacomo, D., Sakarya, B., Gkarlaouni, C.G., Harris, J. \& Storchak, D.A. (2020). ISC-EHB 1964-2016, an Improved Data Set for Studies of Earth Structure and Global Seismicity. Earth and Space Science, 7(1), e2019EA000897. https://doi. org/10.1029/2019EA000897

Engdahl, E.R., van der Hilst, R. \& Buland, R. (1998). Global teleseismic earthquake relocation with improved travel times and procedures for depth determination, Bulletin of the Seismological Society of America, 88(3), 722-743. https://doi.org/10.1785/ BSSA0880030722

Engdahl, E.R. \& Villaseñor, A. (2002). Global Seismicity: 1900-1999. En W.K.H. Lee, H. Kanamori, H., P.C. Jennings \& C. Kisslinger (edit.), International Handbook of Earthquake and Engineering Seismology, Part A, Chapter 41 (pp. 665-690). Academic Press.

Fischer, T. \& Hainzl, S. (2017). Effective Stress Drop of Earthquake Clusters. Bulletin of the Seismological Society of America, 107(5), 2247-2257. https://doi. org/10.1785/0120170035

French, S.W., Warren, L.M., Fischer, K.M., Abers, G.A., Strauch, W., Protti, J.M. \& Gonzalez, V. (2010). Constraints on upper plate deformation in the Nicaraguan subduction zone from earthquake relocation and directivity analysis. Geochemistry, Geophysics, Geosystems, 11(3). https://doi. org/10.1029/2009GC002841

GCMT. (2019). The Global CMT Project. https://www. globalcmt.org/

González, O., Moreno, B. \& Alvarez, L. (2015). La serie de terremotos de El Sauce, septiembre - octubre de 2015. Informe. Fondos de INETER.

Hanks, T.C. \& Kanamori, H. (1979). A moment magnitude scale. Journal of Geophysical Research, 84(B5), 2348-2350. https://doi.org/10.1029/ JB084iB05p02348

Hurukawa, N. \& Imoto, M. (1990). Fine structure of an underground boundary between the Philippine Sea and Pacific plates beneath the Kanto district, Japan. Zisin (Journal of the Seismological Society of Japan), 43, 413-429 (en japonés con resumen en inglés). https://doi.org/10.4294/zisin1948.43.3_413 
Hurukawa, N. \& Imoto, M. (1992). Subducting oceanic crusts of the Philippine Sea and Pacific plates and weak-zone normal compression in the Kanto district Japan. Geophysical Journal International, 109(3), 639-652. https://doi.org/10.1111/j.1365-246X.1992. tb00122.x

INETER. (2014). Estudios especiales del terremoto del 10 de abril y sus réplicas. Boletín mensual de sismos y volcanes. https://webserver2.ineter.gob.ni/sis/ bolsis/bolsis.html

INETER. (2019). Archivos de datos de la red de estaciones de Nicaragua. Fondos de INETER.

ISC. (2020). On-line Bulletin. https://doi.org/10.31905/ D808B830

ISC. (2020a). ISC-EHB dataset. https://doi.org/10.31905/ PY08W6S3

Kanamori, H. \& Kikuchi, M. (1993). The 1992 Nicaragua earthquake: a slow tsunami earthquake associated with subducted sediments. Nature, 361(6414), 714716. https://doi.org/10.1038/361714a0

Kennett, B.L.N., Engdahl, E.R. \& Buland, R. (1995). Constraints on seismic velocities in the Earth from traveltimes. Geophysical Journal International, 122(1), 108-124. https://doi.org/10.1111/j.1365246X.1995.tb03540.x

Kijko,A. (2004). Estimation of the maximum earthquake magnitude, $\mathrm{m}_{\max }$. Pure and Applied Geophysics, 161(8), 1655-1681. https://doi.org/10.1007/s00024004-2531-4

Kijko, A. (2020). Program AUE under Matlab, version 3.01. University of Pretoria, South Africa.

Kijko, A. \& Sellevoll, M.A. (1989). Estimation of earthquake hazard parametersfrom incomplete data files. Part I. Utilization of extreme and complete catalogs with different threshold magnitudes. Bulletin of the Seismological Society of America, 79(3), 645654. https://doi.org/10.1785/BSSA0790030645

Kijko, A. \& Singh, M. (2011). Statistical Tools for Maximum Possible Earthquake Magnitude Estimation. Acta Geophysica, 59(4), 674-700. https:// doi.org/10.2478/s11600-011-0012-6
La Femina, P.C., Connor, C.B., Hill, B.E., Strauch, W. \& Saballos, J.A. (2004). Magma-tectonic interactions in Nicaragua: The 1999 seismic swarm and eruption of Cerro Negro volcano. Journal of Volcanology and Geothermal Research, 137(1-3), 187-199. https://doi. org/10.1016/j.jvolgeores.2004.05.006

Lee, W.H.K., Bennett, R.E. \& Meagher, K.L. (1972). A method of estimating magnitude of local earthquakes from signal duration. USGS Open-File Report 72223, U.S Geological Survey. https://doi.org/10.3133/ ofr 72223

Leeds, D. (1974). Catalogue of Nicaraguan earthquakes. Bulletin of the Seismological Society of America, 64(4), 1135-1158. https://doi.org/10.1785/ BSSA0640041135

Lolli, B., Randazzo, D., Vannucci, G. \& Gasperini, P. (2020). The Homogenized Instrumental Seismic Catalog (HORUS) of Italy from 1960 to Present. Seismological Research Letters, 91(6), 3208-3222. https://doi.org/10.1785/0220200148

Molina, E., Marroquín, G., Escobar, J., Talavera, E., Rojas, W., Climent, A., Camacho, E., Benito, B. \& Lindholm, C. (2008). Proyecto RESIS-II. Evaluación de la amenaza sísmica en Centroamérica. Informe.

Montagner J.P. \& Kennett, B.L.N. (1995). How to reconcile body-wave and normal-mode reference Earth models? Geophysical Journal International, 125(1), 229-248. https://doi.org/10.1111/j.1365246X.1996.tb06548.x

Moreno, B.., González, O., Alvarez, L. \& Palma, M. (2014). Informe del trabajo realizado sobre el terremoto del 10/4/2014 y sus réplicas. Fondos de INETER.

Moreira, A. \& Santos, M.Y. (2007). Concave Hull: A k-nearest neighbours approach for the computation of the region occupied by a set of points. En Proceedings of the Second International Conference on Computer Graphics Theory and Applications - GM/R (pp. 61-68).

NOAA. (1996). The Seismicity Catalog CD-ROM Collection, vol. 1. https:/www.ngdc.noaa.gov/hazard/ data/cdroms/Seismicity_v1/ 
Omori, F. (1894) On the Aftershocks of Earthquakes. Journal of the College of Science, Imperial University of Tokyo, 7, 111-120.

Ottemöller, L., Voss, P. \& Havskov, J. (2018). SEISAN earthquake analysis software for Windows, Solaris, Linux and MacOSX. Version 11.0. http://seisan.info/

Ogata, Y. (1988). Statistical models for earthquake occurrence and residual analysis for point process, Journal of the American Statistical Association, 83(401), 9-27. http://dx.doi.org/10.1080/01621459. 1988.10478560

Ogata, Y. (1999). Seismicity Analysis through Pointprocess Modeling: A Review. through Point-process Modeling: A Review. Pure and applied Geophysics, 155(2-4), 471-507. https://doi.org/10.1007/ s000240050275

Reasenberg, P. (1985). Second-Order Moment of Central California Seismicity, 1969-1982. Journal of Geophysical Research, 90(B7), 5479-5495. https:// doi.org/10.1029/JB090iB07p05479

Reasenberg, P. (2000). Program cluster2000x. https:// earthquake.usgs.gov/research/software/\#CLUSTER

Rokne, J. (1995). The area of a simple polygon. En J. Arvo (Edit.), Graphic Gems II. Academic Press.

Scordilis, E.M. (2006). Empirical global relations converting MS and $\mathrm{mb}$ to moment magnitude. Journal of Seismology, 10(2), 225-236. https://doi. org/10.1007/s10950-006-9012-4

Segura, F.F. (2019). Reseña de la sismicidad en Nicaragua con énfasis en la Depresión Nicaragüense.

Segura, F. \& Alvarez, L. (2016). Serie sísmica de Puerto Morazán. Nicaragua. Boletín Sismos y Volcanes de Nicaragua, junio, 45-53. https://webserver2.ineter. gob.ni/sis/bolsis/bolsis.html

Storchak, D.A., Di Giacomo, D., Engdahl, E.R., Harris, J., Bondár, I., Lee, W.H.K, Bormann, P. \& Villaseñor, A. (2015). The ISC-GEM Global Instrumental Earthquake Catalogue (1900-2009): Introduction. Physics of the Earth and Planetary Interiors, 239, 4863. https://doi.org/10.1016/j.pepi.2014.06.009
Storchak, D.A., Harris, J., Brown, L., Lieser, K., Shumba, B., Verney, R., Di Giacomo, D. \& Korger, E. I. M. (2017). Rebuild of the Bulletin of the International Seismological Centre (ISC), part 1: 1964-1979. Geoscience Letters, 4(32). https://doi.org/10.1186/ s40562-017-0098-Z

Storchak, D.A., Harris, J., Brown, L., Lieser, K., Shumba, B. \& Di Giacomo, D. (2020). Rebuild of the Bulletin of the International Seismological Centre (ISC) part 2: 1980-2010. Geoscience Letters, 7(18). https:// doi.org/10.1186/s40562-020-00164-6

USGS. (2019). Earthquake Hazards Program. Search Earthquake Catalogue. https://earthquake.usgs.gov/ earthquakes/search/

Utheim, T. \& Havskov, J. (2017). SC2SEI. Automatic transfer of phase readings and waveforms from a SeisComp3 data base to a SEISAN data base, Ver. 1.16. ftp://ftp.geo.uib.no/pub/seismo/SOFTWARE/ SC2SEI/sc2sei_v1.00.pdf

Utsu, T. (1961) A Statistical Study on the Occurrence of Aftershocks. The Geophysical Magazine, 30, 521-605.

Utsu, T. (1999). Representation and Analysis of the Earthquake Size Distribution. A Historical Review and Some New Approaches. Pure and applied geophysics. 155(2), 509-535. https://doi.org/10.1007/ s000240050276

Utsu, T., Ogata, Y. \& Matsu'ura, R.S. (1995). The centenary of the Omori formula for a decay law of after-shock activity. Journal of Physics of the Earth, 43(1), 1-33. https://doi.org/10.4294/jpe1952.43.1

Ward, P. L., Harlow, J.D., Gibbs, K. \& Aburto, Q. (1973). Location of the main fault as determined from locations of some aftershocks in Proc. Conf. Managua Earthquake. En C. Rojahn (Ed.), Earthquake Engineering Research Institute (pp. 89-104).

Wells, D. \& Coppersmith, K. (1994). New empirical relationships among magnitude, rupture length, rupture width, rupture area, and surface displacement. Bulletin of the Seismological Society of America, 84(4), 974-1002. https://doi.org/10.1785/ BSSA0840040974 
Wessel, P., \& Smith, W.H.F. (1998): New, improved version of Generic Mapping Tools released, Eos Trans. $A G U$ 79(47), 579. http://dx.doi. org/10.1029/98EO00426

Weston, J., Engdahl, E.R., Harris, J., Di Giacomo, D., \& Storchack, D.A. (2018). ISC-EHB: Reconstruction of a robust earthquake dataset. Geophysical Journal International, 214(1), 474-484. https://doi. org/10.1093/gji/ggy 155

Williams, T \& Kelley, C. (2017): Gnuplot 5.0: An interactive plotting program, manual. http://www. gnuplot.info/docs_5.0/gnuplot.pdf

\section{Agradecimentos}

Se agradece al Dr. Andzrej Kijko, de la Universidad de Pretoria, por facilitar el programa AUE usado para la estimación de los valores de Mmax. Durante los años 2016-2020/02 las actividades fueron financiadas por el INETER (Instituto Nicaragüense de Estudios Territoriales). En el período 2020/03-2021/03 se recibió financiamiento del MTI (Ministerio de Transporte e Infraestructura de Nicaragua) a través del proyecto 2017DEA014 de la AACID. 